\title{
The Quantum Spin Hall Effect: Theory and Experiment
}

\author{
Markus König ${ }^{1}$, Hartmut Buhmann ${ }^{1}$, Laurens W. Molenkamp ${ }^{1}$, \\ Taylor L. Hughes ${ }^{2}$, Chao-Xing Liu ${ }^{3,2}$, Xiao-Liang $\mathrm{Qi}^{2}$ and Shou-Cheng Zhang ${ }^{2}$ \\ ${ }^{1}$ Physikalisches Institut (EP III), Universität Würzburg \\ D-97074 Würzburg, Germany \\ ${ }^{2}$ Department of Physics, McCullough Building, Stanford University \\ Stanford, CA 94305-4045 \\ ${ }^{3}$ Center for Advanced Study, Tsinghua University \\ Beijing, 100084, China
}

February 2, 2008

\begin{abstract}
The search for topologically non-trivial states of matter has become an important goal for condensed matter physics. Recently, a new class of topological insulators has been proposed. These topological insulators have an insulating gap in the bulk, but have topologically protected edge states due to the time reversal symmetry. In two dimensions the helical edge states give rise to the quantum spin Hall (QSH) effect, in the absence of any external magnetic field. Here we review a recent theory which predicts that the QSH state can be realized in $\mathrm{HgTe} / \mathrm{CdTe}$ semiconductor quantum wells. By varying the thickness of the quantum well, the band structure changes from a normal to an "inverted" type at a critical thickness $d_{c}$. We present an analytical solution of the helical edge states and explicitly demonstrate their topological stability. We also review the recent experimental observation of the QSH state in $\mathrm{HgTe} /(\mathrm{Hg}, \mathrm{Cd}) \mathrm{Te}$ quantum wells. We review both the fabrication of the sample and the experimental setup. For thin quantum wells with well width $d_{Q W}<6.3 \mathrm{~nm}$, the insulating regime shows the conventional behavior of vanishingly small conductance at low temperature. However, for thicker quantum wells $\left(d_{Q W}>6.3 \mathrm{~nm}\right)$, the nominally insulating regime shows a plateau of residual conductance close to $2 e^{2} / h$. The residual conductance is independent of the sample width, indicating that it is caused by edge states. Furthermore, the residual conductance is destroyed by a small external magnetic field. The quantum phase transition at the critical thickness, $d_{c}=6.3 \mathrm{~nm}$, is also independently determined from the occurrence of a magnetic field induced insulator to metal transition.
\end{abstract}




\section{Introduction}

Our work on the quantum spin Hall (QSH) effect is motivated both by the quest for spin based electronic devices and by the search for topologically nontrivial states of matter. Unlike logic devices based purely on charge current, quantum spintronic devices integrate information processing and storage units, could operate with low power consumption and perform reversible quantum computations $[1,2]$. Recently, the theoretical prediction of the intrinsic spin Hall effect $[3,4]$ has generated great interest in the field of spintronics, since this effect allows for direct electric manipulation of the spin degrees of freedom without a magnetic field, and the resulting spin current can flow without dissipation. These properties could lead to promising spintronic devices with low power dissipation. The spin Hall effect has been observed recently in both $n[5]$ and $p[6]$ doped semiconductors, however, it is still unclear if the underlying mechanism is intrinsic $[3,4]$ or extrinsic $[7,8]$.

Beyond the potential technological applications, the intrinsic spin Hall effect has guided us in the search for new and topologically non-trivial states of matter. The quantum Hall $(\mathrm{QH})$ state gives the first, and so far the only example of a topologically non-trivial state of matter, where the quantization of the Hall conductance is protected by a topological invariant [9]. Given the fundamental importance of topological quantization in physics, it is highly desirable to search for quantum states of matter characterized by non-trivial topological properties similar to, but distinct from the QH state. Soon after the theoretical prediction of the intrinsic spin Hall effect in doped semiconductors, a rather dramatic prediction was made that the intrinsic spin Hall effect could also be realized in insulators [10]. Subsequently, the QSH state was independently proposed in graphene [11] and in strained semiconductors [12]. The QSH insulator state is invariant under time reversal, has a charge excitation gap in the 2D bulk, but has topologically protected gapless edge states that lie inside the bulk insulating gap. The edge states have a distinct helical property: two states with opposite spin-polarization counter-propagate at a given edge $[11,13,14]$; for this reason, they are also called helical edge states. The edge states come in Kramers' doublets, and time reversal symmetry ensures the crossing of their energy levels at special points in the Brillouin zone. Because of this energy level crossing, the spectrum of a QSH insulator cannot be adiabatically deformed into that of a topologically trivial insulator without helical edge states; therefore, in this precise sense, the QSH insulators represent a topologically distinct new state of matter. The topological properties of the QSH state are mathematically characterized by a $Z_{2}$ topological invariant [15]. States with an even number of Kramers' pairs of edge states at a given edge are topologically trivial, while those with an odd number are topologically non-trivial.

While the initial proposal of the QSH state in graphene [11] provided an interesting theoretical toy model, it was soon shown to be unrealistic since the spin-orbit gap in this system is extremely small $[16,17]$. Bernevig, Hughes and

Zhang [18] initiated the search for the QSH state in semiconductors with an "inverted" electronic gap, and predicted a quantum phase transition in $\mathrm{HgTe} / \mathrm{CdTe}$ 
quantum wells as a function of the thickness $d_{Q W}$ of the quantum well. The quantum well system is predicted to be a conventional insulator for $d_{Q W}<d_{c}$, and a QSH insulator for $d_{Q W}>d_{c}$, with a single pair of helical edge state. In this paper, we review the basic theory of the QSH state in the HgTe/CdTe system, and provide explicit and pedagogical discussion of the helical edge states. We also review recent experimental observations of the QSH effect in this system, through detailed discussions of the sample preparation and the experimental setup.

\section{General Properties of $(\mathrm{Hg}, \mathrm{Cd})$ Te Quantum Wells}

In this section we will review the bandstructure of bulk HgTe and CdTe and present a simple model which is useful in describing the physics of the relevant subbands of a $\mathrm{HgTe} / \mathrm{CdTe}$ quantum well. $\mathrm{HgTe}$ and $\mathrm{CdTe}$ bulk materials have the zinc-blende lattice structure. This lattice has the same geometry as the diamond lattice, i.e., two interpenetrating FCC lattices shifted along the body diagonal, but with a different atom on each sublattice. The presence of two different atoms removes the inversion symmetry of the crystal and reduces the point group symmetry from $O_{h}$ (cubic) to $T_{d}$ (tetrahedral). However, even though the inversion symmetry is explicitly broken, this only has a small effect on the physics. Except for the later discussions on the magneto-resistance in the QSH regime, we will ignore the bulk-inversion asymmetry (BIA).

For both $\mathrm{HgTe}$ and CdTe, the important bands near the typical Fermi level are close to the $\Gamma$-point in the Brillouin zone, and they are the $s$-type band $\left(\Gamma_{6}\right)$ and the $p$-type band which is split to a $J=3 / 2$-band $\left(\Gamma_{8}\right)$ and a $J=1 / 2$-band $\left(\Gamma_{7}\right)$ by spin-orbit coupling. CdTe, as shown in Fig. 1 (a), has a band order similar to GaAs with an $s$-type $\left(\Gamma_{6}\right)$ conduction band and $p$-type valence bands $\left(\Gamma_{8}, \Gamma_{7}\right)$ which are separated from the conduction band by a large energy gap of $\sim 1.6 \mathrm{eV}$. HgTe as a bulk material can be regarded as a symmetry-induced semi-metal. Its negative energy gap of $-300 \mathrm{meV}$ indicates that the $\Gamma_{8}$ band, which usually forms the valence band, is above the $\Gamma_{6}$ band. The light-hole bulk subband of the $\Gamma_{8}$ band becomes the conduction band, the heavy-hole bulk subband becomes the first valence band, and the $s$-type band $\left(\Gamma_{6}\right)$ is pushed below the Fermi level to lie between the heavy-hole subband and the spin-orbit split off band $\left(\Gamma_{7}\right)$. Based on this unusual sequence of the states, such a band structure is called inverted. Due to the degeneracy between the heavy-hole and light-hole bands at the $\Gamma$ point, $\mathrm{HgTe}$ is a zero-gap semiconductor. Note that we have introduced the cubic group representation labels $\Gamma_{6}, \Gamma_{7}, \Gamma_{8}$ for bands near the $\Gamma$-point because various orderings of these bands in the two materials are cumbersome to keep track of and these symbols explicitly convey the symmetry of the bands. We will use these symbols for the remainder of the paper instead of referring to $s$-like, and $p$-like.

When HgTe-based quantum well structures are grown, the peculiar properties of the well material can be utilized to tune the band structure in a unique way. For wide QW layers, the confinement is low and the band structure remains 


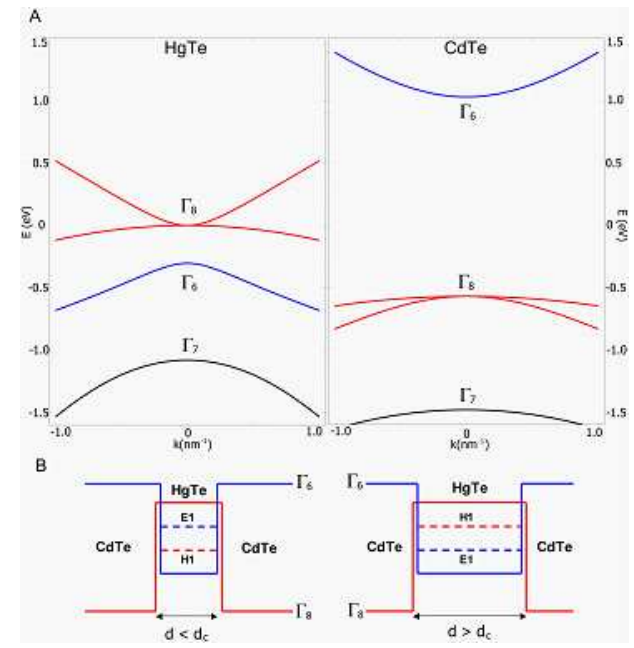

Figure 1: (Upper)Bulk bandstructure for HgTe and CdTe (Lower) Schematic picture of quantum-well geometry and lowest subbands for two different thicknesses.

"inverted". However, the confinement energy increases, when the well width is reduced. Thus, the energy states will be shifted and, eventually, the energy bands will be aligned in a "normal" way, if the QW width falls below a critical size $d_{c}$. We can understand this heuristically as follows: for thin quantum-wells the heterostructure should behave similarly to CdTe and have a normal band ordering, i.e., the bands with primarily $\Gamma_{6}$ symmetry are the conduction subbands and the $\Gamma_{8}$ bands contribute to the valence subbands. On the other hand, as the quantum-well thickness is increased we would expect the material to look more and more like HgTe which has its bands inverted. So as the thickness increases we expect to reach a critical thickness where the $\Gamma_{8}$ and $\Gamma_{6}$ subbands cross and become inverted with the $\Gamma_{8}$ bands becoming conduction subbands and $\Gamma_{6}$ becoming valence subbands. This is illustrated in Fig. 1 (b). The shift of the energy levels with QW width can be seen in Fig. 2, For the band structure, self-consistent Hartree calculations have been performed using a $8 \times 8 \mathbf{~ k} \cdot \mathbf{p}$ model [19]. The notation of the subbands as heavy-hole $(H)$-like and electron $(E)$-like is according to the properties of the respective wave functions [20]. The light-hole-like subbands are energetically remote $(E<-100 \mathrm{meV})$ so that they are not depicted in Fig. 2. The transition from a normal band alignment to an inverted one can clearly be seen in this figure. For a thin QW layer the quantum confinement gives rise to a normal sequence of the subbands, i.e., hole-like bands form the valence band and electron states are in the conduction band. In contrast, a more complex sequence of the energy states is obtained when the QW width $d_{Q W}$ exceeds a critical value $d_{c} \approx 6.3 \mathrm{~nm}$. In this inverted regime, the $H_{1}$ band lies above the $E_{1}$ subband of the valence band and, consequently, is now the lowest conduction subband. We will now develop a simple model and 


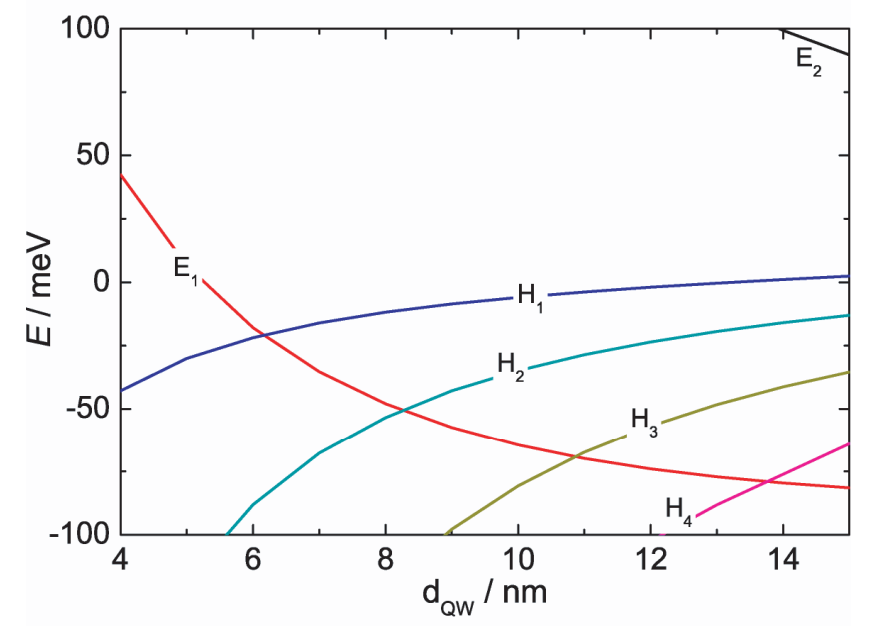

Figure 2: The energy of the states in the quantum well are shown as a function of the width of the HgTe QW layer.

discuss why, immediately after this crossing, we expect to find a time-reversal invariant bulk insulating state with topologically protected edge states, i.e., the QSH state.

\section{Effective Model of the QSHE in $\mathrm{HgTe} / \mathrm{CdTe}$ Quantum Wells}

The effective model of semiconductors whose outer bands are $s$-like and $p$-like near the $\Gamma$-point is the 8-band Kane model [21]. This is a model derived from $\mathbf{k} \cdot \mathbf{p}$ perturbation theory for bulk materials. To calculate the subband spectrum we must write down a Hamiltonian which is inhomogeneous along the quantum well growth direction. In Ref. [18] we used this method to derive an effective 4-band model for the subbands that participate in the inversion crossing. Rather than repeat this calculation we will give some simple symmetry arguments about the form of the model. Under the cubic symmetry group the relevant $\Gamma_{6}$-band transforms antisymmetrically under "cubic"-parity because it is an anti-bonding band (in CdTe and $\mathrm{HgTe}$ ), even though it has an $s$-wave like character. The $\Gamma_{8}$ band transforms symmetrically since it is a bonding band. The "conduction" subbands $E_{1}, E_{2} \ldots$ and heavy-hole $H_{1}, H_{2} \ldots$ subbands all alternate their parity under $z \rightarrow-z$ inversion. For our purposes we neglect the bonding combination of the $\Gamma_{6}$ and light-hole bands whose subbands are denoted $L_{1}, L_{2} \ldots$ because these bands are well-separated in energy from the bands in which we are interested. We will be focussed on only the lowest subbands and as mentioned before, the $E_{1}$ subband actually contains components of the $\Gamma_{6}$ band 
and the $\Gamma_{8}$ light-hole band i.e. $\left|E_{1}, \pm 1 / 2\right\rangle=\alpha(z)\left|\gamma^{6}, \pm 1 / 2\right\rangle+\beta(z)\left|\gamma^{8}, \pm 1 / 2\right\rangle$. In order to preserve the transformation of the $E_{1}$ subbands under inversion of the growth (z)-direction the envelope function $\alpha(z)$ must be even under $z \rightarrow-z$ while the envelope function $\beta(z)$ piece must be odd.

The relevant subbands, $E_{1}$ and $H_{1}$, under our assumption of inversion symmetry, must be doubly degenerate since time-reversal symmetry is present. We will label the states with the basis $\left|E_{1}+\right\rangle,\left|E_{1}-\right\rangle,\left|H_{1}+\right\rangle,\left|H_{1}-\right\rangle$, where $\left|E_{1} \pm\right\rangle$ and $\left|H_{1} \pm\right\rangle$ are the two sets of Kramers' partners. The states $\left|E_{1} \pm\right\rangle$ and $\left|H_{1} \pm\right\rangle$ have opposite transformations under parity, so a Hamiltonian matrix element that connects them must be odd under parity since we assumed our Hamiltonian preserves inversion symmetry. Thus, to lowest order in $k,\left(\left|E_{1}+\right\rangle,\left|H_{1}+\right\rangle\right)$ and $\left(\left|E_{1}-\right\rangle,\left|H_{1}-\right\rangle\right)$ will each be coupled generically via a term linear in $k$. The $\left.\left|H_{1}+\right\rangle\right)$ heavy-hole state is formed from the spin-orbit coupled p-orbitals $\left.\left|p_{x}+i p_{y}, \uparrow\right\rangle\right)$, while the $\left.\left|H_{1}-\right\rangle\right)$ heavy-hole state is formed from the spin-orbit coupled p-orbitals $\left.\left|-\left(p_{x}-i p_{y}\right), \downarrow\right\rangle\right)$. Therefore, to preserve rotational symmetry around the growth-axis (z-axis) the matrix elements must by proportional to $k_{ \pm}=k_{x} \pm i k_{y}$. The only terms allowed in the diagonal elements are terms that have even powers of $k$ including $k$-independent terms. The subbands must come in fully degenerate pairs so there can be no matrix elements between the + state and the - state of the same band. Finally, we note that if non-zero matrix elements coupled $\left|E_{1}+\right\rangle,\left|H_{1}-\right\rangle$ or $\left|E_{1}-\right\rangle,\left|H_{1}+\right\rangle$ then this would induce a higher-order process that would couple the \pm states of the same band, thus splitting the degeneracy. So these matrix elements are forbidden as well. These simple arguments lead us to the following model

$$
\begin{aligned}
\mathcal{H} & =\left(\begin{array}{cc}
h(k) & 0 \\
0 & h^{*}(-k)
\end{array}\right) \\
h(k) & =\epsilon(k) \mathrm{I}_{2 \times 2}+d_{a}(k) \sigma^{a} \\
\epsilon(k) & =C-D\left(k_{x}^{2}+k_{y}^{2}\right) \\
d_{a}(k) & =\left(A k_{x},-A k_{y}, M(k)\right) \\
M(k) & =M-B\left(k_{x}^{2}+k_{y}^{2}\right) .
\end{aligned}
$$

where we have used the basis order $\left(\left|E_{1}+\right\rangle,\left|H_{1}+\right\rangle,\left|E_{1}-\right\rangle,\left|H_{1}-\right\rangle\right), A, B, C, D, M$ are material parameters that depend on the quantum well geometry, and we have chosen the zero of energy to be the valence band edge of $\mathrm{HgTe}$ at $\mathbf{k}=0$ as in Fig. 1. $\mathcal{H}$ is equivalent to two copies of the massive Dirac Hamiltonian but with a $k$-dependent mass $M(k)$.

For the purposes of studying the topological properties of this system, as well as the edge states, we will work with a lattice regularization of this model which will give us the dispersions over the entire Brillouin zone torus, i.e., a simplified tight-binding representation. Since all of the interesting physics at low energy occurs near the $\Gamma$-point, the behavior of the dispersion at high-energy is not important. Thus, we can choose a regularization to make our calculations 
simple. This simplified lattice model is

$$
\begin{aligned}
\mathcal{H} & =\left(\begin{array}{cc}
h(k) & 0 \\
0 & h^{*}(-k)
\end{array}\right) \\
h(k) & =\epsilon(k) \mathrm{I}_{2 \times 2}+d_{a}(k) \sigma^{a} \\
\epsilon(k) & =C-2 D a^{-2}\left(2-\cos \left(k_{x} a\right)-\cos \left(k_{y} a\right)\right) \\
d_{a}(k) & =\left(A a^{-1} \sin \left(k_{x} a\right),-A a^{-1} \sin \left(k_{y} a\right), M(k)\right) \\
M(k) & =M-2 B a^{-2}\left(2-\cos \left(k_{x} a\right)-\cos \left(k_{y} a\right)\right) .
\end{aligned}
$$

It is clear that near the $\Gamma$-point the lattice Hamiltonian reduces to the $\mathbf{k} \cdot \mathbf{p}$ Hamiltonian in Eq. 1. For simplicity, below we take the lattice constant $a=1$, which corresponds to a redefinition of the units.

The bulk band structure of this model has the energy spectrum

$$
\begin{aligned}
E_{ \pm} & =\epsilon(k) \pm \sqrt{d_{a} d_{a}} \\
& =\epsilon(k) \pm \sqrt{A^{2}\left(\sin ^{2} k_{x}+\sin ^{2} k_{y}\right)+M^{2}(k)} .
\end{aligned}
$$

This spectrum gives a bulk insulator as long as $\sqrt{d_{a} d_{a}} \neq 0$ (and no indirect gap is formed) and there is a gapless spectrum at a phase transition critical point when this quantity vanishes. The phase transition and topological properties do not depend on $\epsilon(k)$ so we will ignore this term for now. The lattice Hamiltonian has several critical points, i.e., several points in $\left(k_{x}, k_{y}, M\right)$-space where $d_{a}=0$, but there is only one that occurs in the low-energy spectrum near the $\Gamma$-point. Since the model is only valid for small $k$ we will confine ourselves to this single critical point. We can see from the form of $d_{a}$ that this critical point will occur at $k_{x}=k_{y}=0$ and $M=0$ (assuming $B$ is finite). For $M$ close to 0 , the gap is minimal around $\Gamma$ point, and the system is described by two flavors of Dirac fermions with mass $M$ and $-M$. The Hall conductance of the massive Dirac model jumps by a quantum $\left(e^{2} / h\right)$ when the mass changes sign [22]. That is, $\sigma_{H}\left(M \rightarrow 0^{+}\right)-\sigma_{H}\left(M \rightarrow 0^{-}\right)=e^{2} / h$. For each $2 \times 2$ block of the lattice model (Eq. 6), the Hall conductance can also be calculated explicitly through the Kubo formula, which leads to $\sigma_{H}=c_{1} e^{2} / h$, with $c_{1} \in \mathrm{Z}$ the following Pontryagin winding number $[23,24]$ :

$$
c_{1}=-\frac{1}{8 \pi^{2}} \int \frac{d^{2} k}{(2 \pi)^{2}} \epsilon_{a b c} \epsilon_{i j} \frac{d_{a} \partial_{i} d_{b} \partial_{j} d_{c}}{d^{3}}
$$

Direct numerical calculations show that $c_{1}=0$ for $M / 2 B<0$ and $c_{1}= \pm 1$ for $0<M / 2 B<2$, where $+(-)$ corresponds to the $h(k)\left(h^{*}(-k)\right)$ block. Consequently, the system with $M / 2 B>0$ is equivalent to two independent quantum Hall systems with opposite Hall conductance, which guarantees a pair of counter propagating edge states on each boundary of the system. (The condition $M<4 B$ is always satisfied for the physical system since the gap $2 M$ is always small.) Once such a pair of edge states is established on the boundary they are robust under time-reversal invariant perturbations, and are protected 
by time-reversal symmetry which generates a $Z_{2}$ topological quantum number. In other words, the system (Eq. 6) with $M / 2 B>0$ is a topologically nontrivial insulator which cannot be adiabatically tuned to a trivial insulator. More discussions about the topological stability of this system will be presented in the next section.

So far we have developed a simple 4-band lattice model for the $E_{1}$ and $H_{1}$ subbands and studied the properties of the bulk band structure and the lowenergy critical point. We will now solve the model in a finite strip geometry to explicitly show the existence of topologically protected edge states for the nontrivial regime. Since the identity term $\epsilon(k)$ in the Hamiltonian (Eq. 6) provides only an overall energy shift to all the states without changing the wavefunction profiles, we will omit this term below. The Hamiltonian (Eq. 6) can be rewritten as

$$
\mathcal{H}=\sum_{\mathbf{k}}\left(A \sin \left(k_{x}\right) \Gamma^{1}+A \sin \left(k_{y}\right) \Gamma^{2}+\mathcal{M}(\mathbf{k}) \Gamma^{5}\right) c_{\mathbf{k}}^{\dagger} c_{\mathbf{k}}
$$

where $\Gamma^{a}, a=1 \ldots 5$ form a Clifford algebra and $\mathcal{M}(\mathbf{k})$ is defined in Eq. 6. For this model we have $\Gamma^{1}=\sigma^{x} \otimes s^{z}, \Gamma^{2}=-\sigma^{y} \otimes 1, \Gamma^{3}=\sigma^{x} \otimes s^{x}, \Gamma^{4}=\sigma^{x} \otimes s^{y}, \Gamma^{5}=$ $\sigma^{z} \otimes 1$ where $\sigma^{i}$ acts on the $\left(E_{1}, H_{1}\right)$ space and $s^{i}$ acts on $(+,-)$ space. As stated above, around the $\Gamma$-point in the Brillouin zone this Hamiltonian behaves as a massive Dirac Hamiltonian in 2-d which means that for non-zero $M$ the bulk spectrum will be gapped. Now we want to show that for certain values of the parameter $M$ the system is in a topological insulator state characterized by a bulk energy gap and edge-states which lie in the gap. In order to study the edge-state spectrum we must first pick a particular edge. Since this simplified model has square lattice symmetry we pick the edges to be perpendicular to the $y$-axis. This implies that $k_{y}$ is no longer a good quantum number since the system is not homogeneous in this direction, but instead has boundaries on the lines $y=0, L$. We must Fourier transform $k_{y}$ into position space to obtain a 1 -d tight-binding model which depends on the other momentum $k_{x}$. We make the substitution

$$
c_{\mathbf{k}}=\frac{1}{L} \sum_{j} e^{i k_{y} j} c_{k, j}
$$

where $k \equiv k_{x}$. This gives the Hamiltonian:

$$
\begin{aligned}
\mathcal{H} & =\sum_{k, j}\left(\mathcal{M} c_{k, j}^{\dagger} c_{k, j}+\mathcal{T} c_{k, j}^{\dagger} c_{k, j+1}+\mathcal{T}^{\dagger} c_{k, j+1}^{\dagger} c_{k, j}\right) \\
\mathcal{M} & =A \sin \left(k_{x}\right) \Gamma^{1}-2 B\left[2-M / 2 B-\cos \left(k_{x}\right)\right] \Gamma^{5} \\
\mathcal{T} & =\frac{i A}{2} \Gamma^{2}+B \Gamma^{5}
\end{aligned}
$$

We expect edge states to be exponentially localized on the boundary so we look for solutions with the following ansatz $[25,26]$

$$
\psi_{\alpha}(j)=\lambda^{j} \phi_{\alpha}
$$


where $\lambda$ is a complex number, $j$ is the index of the $j$-th lattice site in the $y$ direction which runs from $0 \ldots L$, and $\phi_{\alpha}$ is a constant 4-component spinor with $\alpha=1 \ldots 4$. Thus $H \psi_{\alpha}=E \psi_{\alpha}$ gives

$$
\lambda^{-1} \mathcal{T}_{\alpha \beta} \phi_{\beta}+\lambda \mathcal{T}_{\alpha \beta}^{\dagger} \phi_{\beta}+\mathcal{M}_{\alpha \beta} \phi_{\beta}=E \phi_{\beta} .
$$

This Hamiltonian is particle-hole symmetric, and time-reversal symmetric. Thus, at non-zero $M$ a gap is opened around $E=0$ and at $k_{x}=0$ we expect the edge states to have energy $E=0$. So, at the $\Gamma$-point we generate the following simple equation

$$
\left[\frac{i A}{2}\left(\lambda^{-1}-\lambda\right) \Gamma^{2}+B\left(\lambda+\lambda^{-1}\right) \Gamma^{5}+\mathcal{M}(0) \Gamma^{5}\right] \phi=0 .
$$

Multiplying both sides by $\Gamma^{5}$ gives

$$
\frac{A}{2}\left(\lambda^{-1}-\lambda\right)\left(i \Gamma^{5} \Gamma^{2}\right) \phi=-\left(B\left(\lambda+\lambda^{-1}\right)+\mathcal{M}(0)\right) \phi
$$

The operator $i \Gamma^{5} \Gamma^{2}$ has eigenvalues \pm 1 . First consider $i \Gamma^{5} \Gamma^{2} \phi=-\phi$, under which condition Eq. 18 becomes a quadratic equation for $\lambda$ which is easily solved to give

$$
\lambda_{(1,2)}=\frac{-\mathcal{M}(0) \pm \sqrt{\mathcal{M}^{2}(0)+\left(A^{2}-4 B^{2}\right)}}{A+2 B} .
$$

It is trivial to see that if $\lambda$ is a solution for $i \Gamma^{5} \Gamma^{2} \phi=-\phi$ then $\lambda^{-1}$ is a solution for the opposite eigenvalue $i \Gamma^{5} \Gamma^{2} \phi=\phi$. Denoting $\phi_{s+(-)}$, with $s=1,2$ being the two eigenstates of $i \Gamma^{5} \Gamma^{2}$ with eigenvalue $+1(-1)$, then a generic solution of the Schroedinger equation can be written as

$$
\psi(j)=\sum_{s}\left(a_{s} \lambda_{(1)}^{j}+b_{s} \lambda_{(2)}^{j}\right) \phi_{s+}+\sum_{s}\left(c_{s} \lambda_{(1)}^{-j}+d_{s} \lambda_{(2)}^{-j}\right) \phi_{s-}
$$

The open boundary condition can be expressed as $\psi_{\alpha}(j=0)=0$. Since $\phi_{s \pm}$ are mutually orthogonal, this condition leads to $a_{s}+b_{s}=0, c_{s}+d_{s}=0$. On the other hand, the requirement of normalizability requires that only the exponentially decaying terms be included in $\psi(j)$. Consequently, a normalizable edge state solution exists only when $\left|\lambda_{(1)}\right|<1,\left|\lambda_{(2)}\right|<1$ (with $c_{s}=d_{s}=0$ ) or $\left|\lambda_{(1)}\right|>1,\left|\lambda_{(2)}\right|>1$ (with $\left.a_{s}=b_{s}=0\right)$.

Such a derivation can be easily generalized to $k_{x} \neq 0$ case. Since $\left[i \Gamma^{5} \Gamma^{2}, \Gamma^{1}\right]=$ 0 the term $A \sin \left(k_{x}\right) \Gamma^{1}$ can be simultaneously diagonalized to add to the energy. The term involving $\cos \left(k_{x}\right)$ simply contributes to the mass term. It is convenient to choose the eigenstates $\phi_{s \pm}$ so that $\Gamma^{1} \phi_{s \pm}=s \phi_{s \pm}$, in which condition the two edge state wavefunctions $\psi_{s}(j)$ and corresponding energy $E_{s}\left(k_{x}\right)$ are 
written as

$$
\begin{aligned}
\psi_{s}(j) & =\sum_{s}\left(\lambda_{(1)}^{j}-\lambda_{(2)}^{j}\right) \phi_{s+} \\
E_{s}\left(k_{x}\right) & =-A s \sin \left(k_{x}\right) \\
\text { with } \lambda_{(1,2)} & =\frac{-m(k, M) \pm \sqrt{m(k, M)^{2}+\left(A^{2}-4 B^{2}\right)}}{A+2 B} \\
m(k, M) & =-2 B\left(2-M / 2 B-\cos \left(k_{x}\right)\right) .
\end{aligned}
$$

To have a normalizable solution we still must have $\left|\lambda^{(1,2)}\right|<1$ or $\left|\lambda^{(1,2)}\right|>1$, which leads to

$$
-2 B<m(k, M)<2 B
$$

In other words, for a fixed $M$ an edge state will exist only for the finite region of $k$ that satisfies this inequality.

For the dispersion in Eq. 22 we can see that two edge states with opposite $\Gamma^{1}$ eigenvalue are propagating in opposite directions. At $k_{x}=0$, the edge states exist for $0<M<4 B$, that is, $m(0, M)$ satisfies the inequality in Eq. 23 for $M / 2 B \in(0,2)$. When $k_{x}$ is varied from 0 to $\pi$, the edge states are dispersive until the inequality (Eq. 23) is violated at some finite $k_{x}$, which, physically, is the wavevector where the edge states touch the bulk (extended) states. In Fig. 3 we have plotted the energy spectrum as a function of $k_{x}$ from (numerical) exact diagonalization, and from our analytic solution. In our analytic solution, and with fixed $M$, we solve for the $k_{x}$-points where the edge state solution is no longer normalizable, which occurs when the edge state becomes degenerate with the bulk states. It is at these $k$-points where the edge state dispersion enters the bulk and is no longer visible.

\section{Topological Properties of the Helical Liquid}

Similar to the case of quantum Hall effect, the topological properties of the QSH system can be understood by studying the low energy effective theory of the edge states. The edge states of the quantum Hall effect are described by a chiral Luttinger liquid theory [27]. Such a chiral theory only contains, say, left movers, so that the current carried by the edge excitations cannot be destroyed by any perturbation due to the absence of counter-propagating modes in which to backscatter. In comparison, the effective theory of the quantum spin Hall edge states do contain both left and right movers, and can be written as

$$
H=\int \frac{d k}{2 \pi}\left(\psi_{k+}^{\dagger} v k \psi_{k+}-\psi_{k-}^{\dagger} v k \psi_{k-}\right)+H_{\mathrm{pert}}
$$

where $H_{\text {pert }}$ represents possible perturbation terms, including disorder and/or electron-electron interactions.

If time-reversal symmetry is not present, a simple "mass" term can be added in $H_{\text {pert }}$ so that the spectrum becomes gapped:

$$
H_{\text {mass }}=\int \frac{d k}{2 \pi} m\left(\psi_{k+}^{\dagger} \psi_{k-}+\text { h.c. }\right)
$$




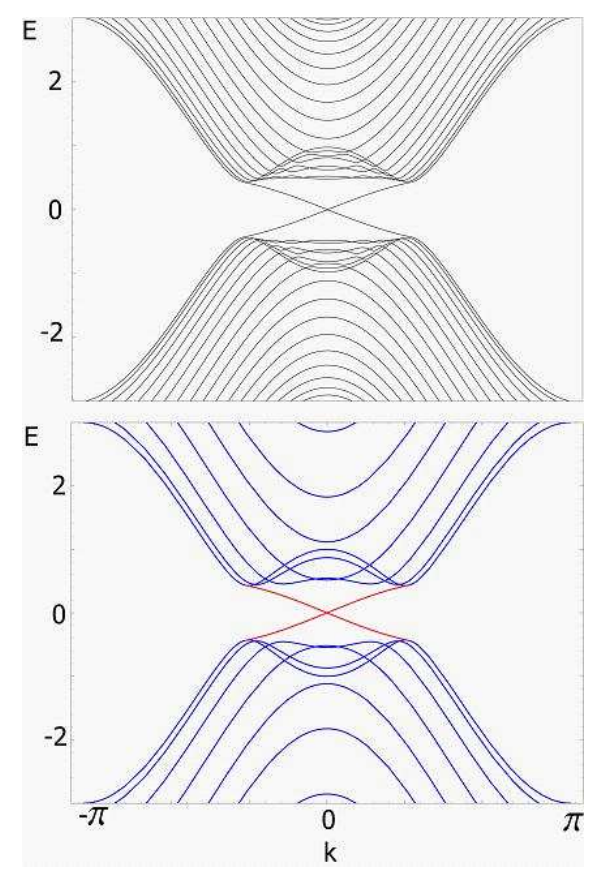

Figure 3: (Upper)Exact diagonalization of 1-d tight binding model. (Lower) analytic solution for bulk (blue) and edge states(red). Bulk bands are plotted vs $k_{x}$ for several different values of $k_{y}$ in order to reproduce the look of the exact diagonalization results.

However, the time-reversal symmetry of the electron system is expressed as

$$
T^{-1} \psi_{k+} T=\psi_{-k,-}, T^{-1} \psi_{k-} T=-\psi_{-k,+}
$$

which implies

$$
T^{-1} H_{\text {mass }} T=-H_{\text {mass }} .
$$

Consequently, $H_{\text {mass }}$ is a time-reversal symmetry breaking perturbation. More generally, if we define the "chirality" operator

$$
C=N_{+}-N_{-}=\int \frac{d k}{2 \pi}\left(\psi_{k+}^{\dagger} \psi_{k+}-\psi_{k-}^{\dagger} \psi_{k-}\right),
$$

any operator that changes $C$ by $2(2 n-1), n \in \mathrm{Z}$ is time-reversal odd. In other words, time-reversal symmetry requires that the Hamiltonian $H_{\text {pert }}$ only include processes of $2 n$ particles back scattering, such as $\psi_{k+}^{\dagger} \psi_{k^{\prime}+}^{\dagger} \psi_{p-} \psi_{p^{\prime}-}$. Therefore, the most relevant perturbation in normal metals, i.e., $\psi_{k+}^{\dagger} \psi_{k^{\prime}-}$, is forbidden by time-reversal symmetry. This is essential for the topological stability of the edge states. This edge state effective theory is thus non-chiral and is qualitatively 
different from the usual spinless or spinful Luttinger liquid theories. It can be considered as aa example of a new class of one-dimensional critical theories, describing a "helical liquid" $[13,14]$. Specifically, for the non-interacting case no time-reversal invariant perturbation is available to induce back scattering, so that the edge state is robust under any disorder.

(a)

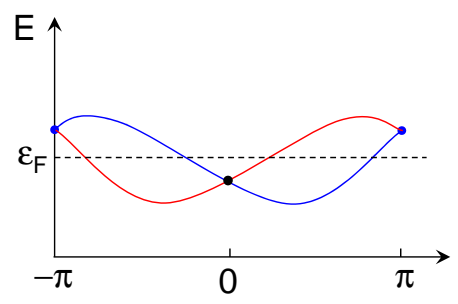

(b)

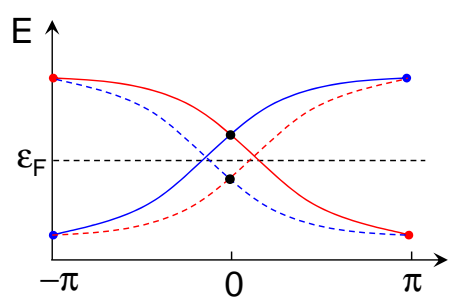

Figure 4: (a) The energy dispersion of a one-dimensional time-reversal invariant system. Kramer's degeneracy is required at $k=0$ and $k=\pi$, so that the energy spectrum always crosses the Fermi level $\epsilon_{F} 4 n$ times. (b) The energy dispersion of the helical edge states on one boundary of the QSH system (solid lines). At $k=0$ the edge states are Kramers' partners of each other, while at $k=\pi$ they merge to the bulk and pair with the edge states of the other boundary (dash lines). In both (a) and (b), red and blue lines form Kramers' partners of each other.

Now consider the case with two flavors of helical edge states on the boundary, i.e., a 1-d theory with two left movers and two right movers, with the Hamiltonian

$$
H=\int \frac{d k}{2 \pi} \sum_{s=1,2}\left(\psi_{k s+}^{\dagger} v_{s} k \psi_{k s+}-\psi_{k s-}^{\dagger} v_{s} k \psi_{k s-}\right) .
$$

Due to the same reason as the one-flavor case, the single particle back scattering terms between Kramers' doublets $\psi_{k s+}^{\dagger} \psi_{-k, s-}$ is forbidden. However, the back scattering between different flavors such as $\psi_{k 1+}^{\dagger} \psi_{-k, 2-}$ can be non-vanishing, which makes such a theory qualitatively equivalent from a spinful Luttinger liquid with four degrees of freedom. A mass term like $\int \frac{d k}{2 \pi} M\left(\psi_{k 1+}^{\dagger} \psi_{k 2-}+\right.$ h.c. $)$ can immediately open a gap in the system, which implies that the existence of gapless edge states is not generic, as shown in Fig. 5. In other words, two copies of the helical liquid becomes a topologically trivial theory. More generally, an edge system with time-reversal symmetry is a nontrivial helical liquid when there is an odd number of left(or right) movers, and trivial when there is an even number. Such a property makes it natural to characterize the topology in the QSH systems by a $Z_{2}$ topological quantum number. 

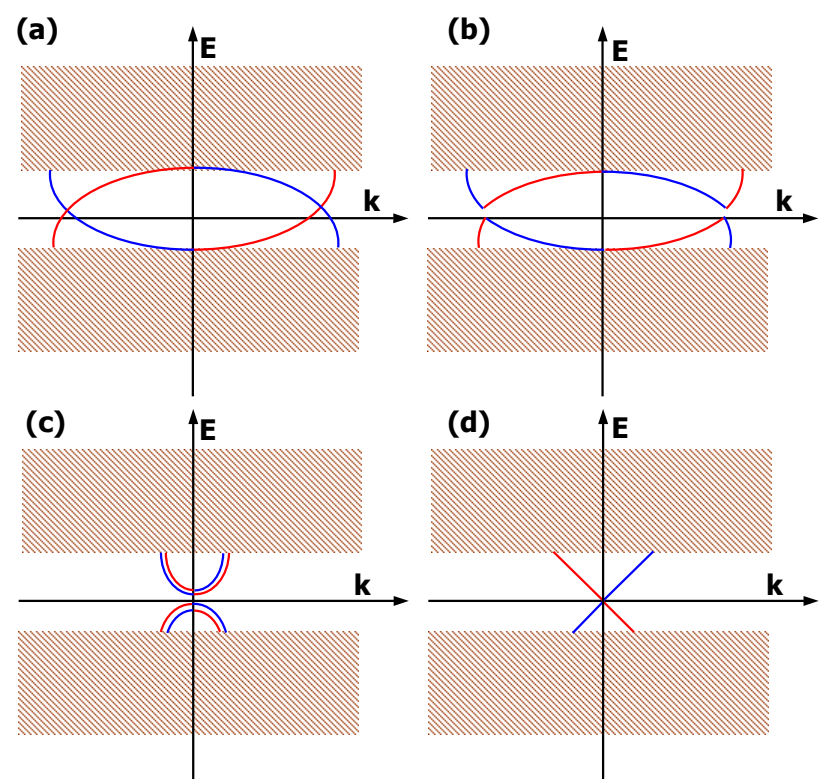

Figure 5: Brown areas are regions of bulk states. Red and blue dispersing edge states represent Kramers' partners (a)An even number of pairs of fermion branches crossing at $k$ points which do not transform to themselves under timereversal (b) A slight perturbation added to the system causes the edge states in (a) to form a gap. Kramers' theorem is still satisfied but these edge states are unstable to gap formation (c) Two pairs of fermion branches that initially crossed at the special point $\mathbf{k}=0$ are shown after an infinitesimal perturbation is added. A gap is formed and Kramers' theorem is satisfied but this configuration is also unstable to gap formation (d)A single pair of fermion branches crosses at $\mathbf{k}=0$. A perturbation cannot open a gap because in that case there would be two states which were singly degenerate which will not satisfy Kramers' theorem, thus this configuration is stable.

There is also an alternative way to understand the qualitative difference between even and odd branches, which is expressed as a "no-go" theorem in Ref. 13: There is always an even number of Kramers' pairs at the Fermi energy for an arbitrary one-dimensional band structure. Such a no-go theorem is a time-reversal invariant analog of the Nielsen-Ninomiya no-go theorem for chiral fermions on a lattice $[28,29]$. For spinless fermions there are always an equal number of left movers and right movers at the Fermi level, which leads to the fermion doubling problem in odd spatial dimensions. Similarly, for a time-reversal symmetric system with half-odd integer spin, Kramers' theorem requires each energy band to be accompanied by its Kramers' partner, so that the number of low energy channels are doubled again. As shown in Fig. 目 (a), a Kramers' pair of states at $k=0$ must re-form into pairs with each other when $k$ goes from 0 to $\pi$ and $2 \pi$, which requires the bands to cross the Fermi level $4 n$ 
times. Seemingly disheartening, there is actually an exception to this theorem analogous to the reason why a chiral liquid exists in the quantum Hall effect. The exception is that a helical liquid with an odd number of fermion branches can occur if it is holographic, i.e., appears at the boundary (edge) of a $2 \mathrm{~d}$ bulk system. As shown in Fig. 3, in this case the edge states are Kramers' partners of each other at $k=0$, but they merge into bulk states at some finite $k_{c}$, so that they don't have to be paired with each other at $k=\pi$. More accurately, at $k>k_{c}$ the edge states on both the left and right boundaries become bulk states at $k>k_{c}$, and form a Kramers' pair with each other, as shown in Fig. 4(b).

The no-go theorem also provides a physical understanding of the topological stability of the helical liquid. Any local perturbation on the boundary of a 2d QSH system can be considered as coupling a "dirty surface layer" with the unperturbed helical edge states. Whatever perturbation is considered, the "dirty surface layer" is always a one-dimensional system, so that there are always an even number of Kramers' pairs of low energy channels. Since the helical liquid has only an odd number of Kramers' pairs, the coupling between them can only annihilate an even number of Kramers' pairs, so that at least one-pair of gapless edge states can survive.

Similar to the correspondence of chiral edge states and bulk Chern number in quantum Hall effect [9], the $Z_{2}$ topological stability of helical edge states can be determined by a $Z_{2}$ topological quantum number defined in the two-dimensional Brillouin zone, as shown in Refs. 15 and 30. Several alternative ways to define the $Z_{2}$ invariant and its generalization to three-dimensional topological insulators were also proposed in the literature [31-36]. In the quantum Hall case, the Chern number can be defined by introducing twisted boundary conditions, even when disorder and interactions are present so that momentum is not good quantum number [37]. Similar twisted boundary conditions can be introduced to define the $Z_{2}$ invariant when disorder is considered [38]. However, the generalization to the many-body case with interactions is not so straightforward, since the Kramers' degeneracy is only defined for a single electron state. Some efforts have been made toward this direction recently [39].

In summary, in this section we have discussed the topological stability of the edge states, which is essential for the quantum spin Hall effect in HgTe quantum wells to be realized in an experimental system. Although the edge states are non-chiral, one can still detect them through the longitudinal conductance it contributes. In the ballistic limit, a helical liquid provides a metallic onedimensional channel, which leads to a conductance of $e^{2} / h$. For a system with two boundaries the net longitudinal conductance will be $2 e^{2} / h$. In the rest of the paper we will review the experimental observations of the transport properties of $\mathrm{HgTe}$ quantum wells and present theoretical analysis of the results.

\section{Quantum Well Fabrication}

As discussed above, we anticipate that the transition from a normal to an inverted band structure in $\mathrm{HgTe}$ QWs coincides with a phase transition from a 
trivial insulator to the QSH insulator. Experimentally, we have investigated the transport properties of $\mathrm{HgTe}$ samples with QW width in the range from $4.5 \mathrm{~nm}$ to $12.0 \mathrm{~nm}$, so as to cover both the normal and the inverted band structure regime. The samples were grown by molecular beam epitaxy (MBE) and the layer sequence is schematically depicted in Fig. 6. The wells are sandwiched by $\mathrm{Hg}_{0.3} \mathrm{Cd}_{0.7} \mathrm{Te}$ barriers and $n$-type modulation doped using I-doping on both sides of the QW layer. Recent advances in the growth of HgTe-based QW struc-

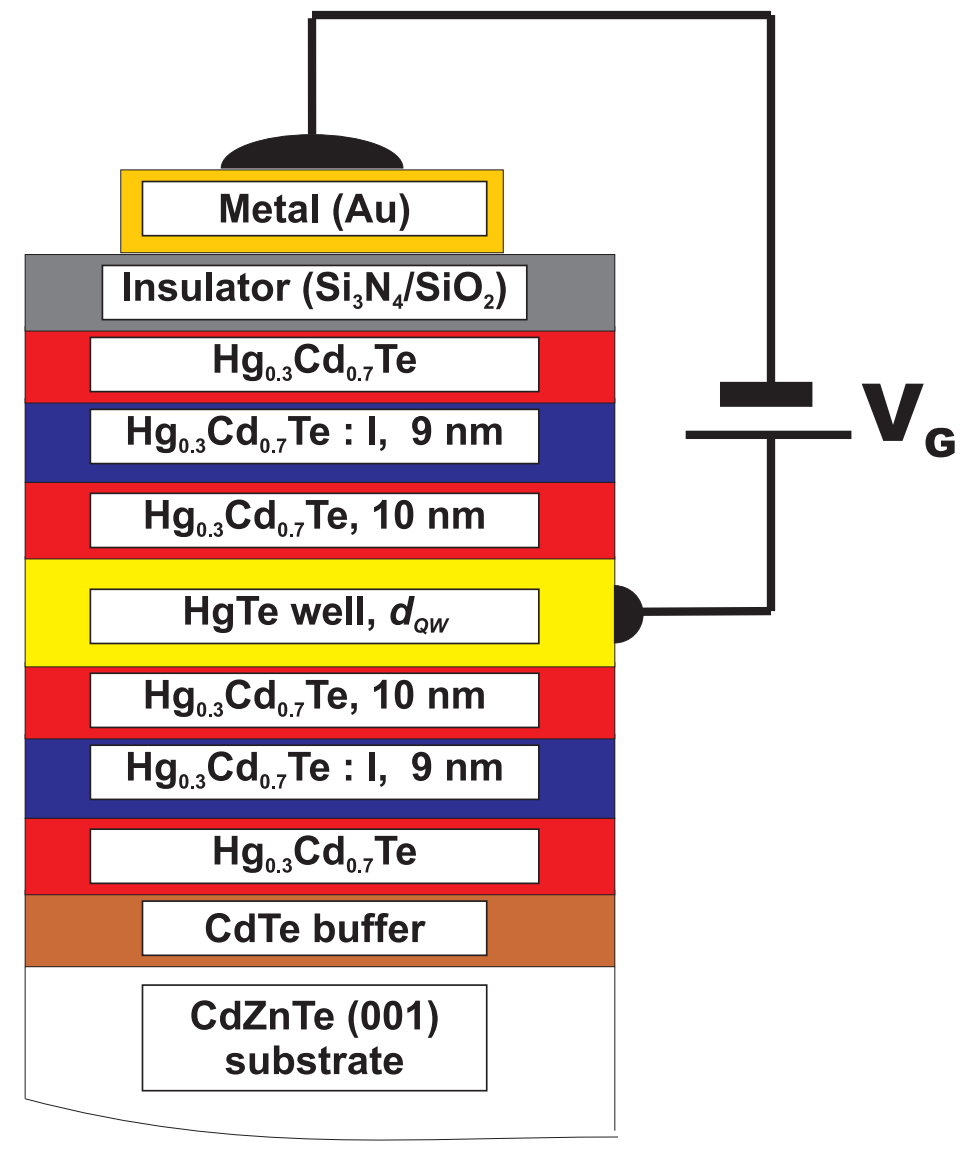

Figure 6: Schematics of the layer sequence of the MBE-grown quantum well structures.

tures, that were incorporated in these structures are documented in Ref. [40]. For example, increasing the spacer width between the quantum well and the doping layer results in an enhancement of the carrier mobility $\mu$, and samples with mobilities of several $10^{5} \mathrm{~cm}^{2} /(\mathrm{Vs})$ even at low densities $n<5 \times 10^{11} \mathrm{~cm}^{-2}$ have been used for the actual measurements. In such samples, the elastic mean free path is of the order of several microns.

The devices have been structured by means of optical and electron beam (e- 
beam) lithography. So as to avoid $\mathrm{Hg}$ evaporation, the $\mathrm{HgTe}$ layer has to be grow at temperatures as low as $180^{\circ} \mathrm{C}$ and the sample has to be kept well below this temperature during the entire process of fabrication. Standard processes based on PMMA (polymethylmethacrylate) as a resist can not be used, since these usually demand temperatures up to $200^{\circ} \mathrm{C}$ for bake-out. While we fabricated our first $\mathrm{HgTe}$-based nanostructures using a standard photoresist for the e-beam steps [41], we have, for the samples discussed here, employed a dedicated low temperature PMMA technology. In combination with Ar-ion etching we can reproducibly fabricate structures with dimensions down to ca. $100 \mathrm{~nm}$.

For the investigation of the QSH effect, devices in a Hall bar geometry of various dimensions have been fabricated from $\mathrm{QW}$ structures with a $\mathrm{QW}$ width of $4.5 \mathrm{~nm}, 5.5 \mathrm{~nm}, 6.4 \mathrm{~nm}, 6.5 \mathrm{~nm}, 7.2 \mathrm{~nm}, 7.3 \mathrm{~nm}, 8.0 \mathrm{~nm}$ and $12.0 \mathrm{~nm}$, respectively. The layout of the devices is sketched in Fig. 7 .

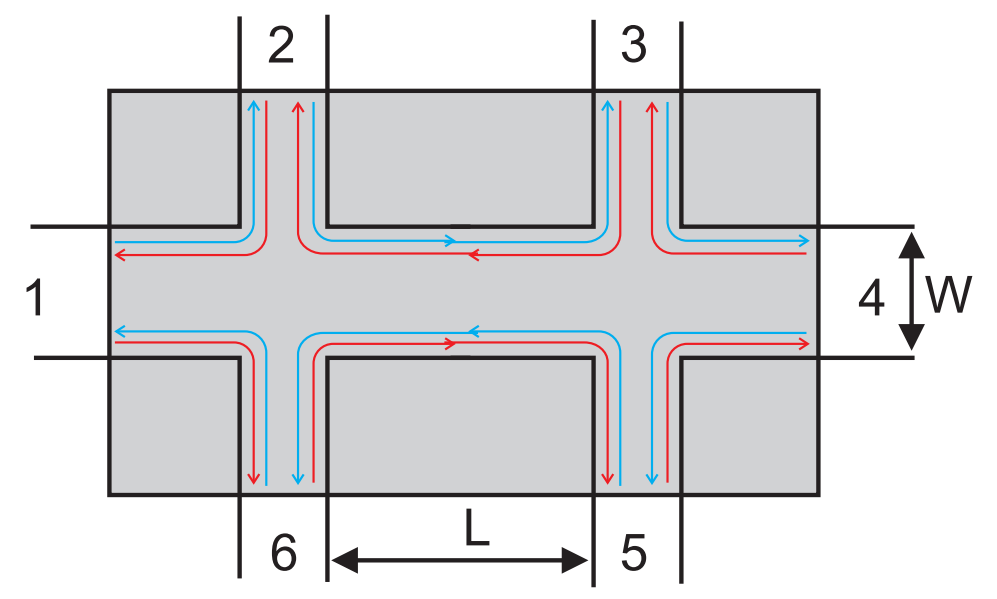

Figure 7: This schematic sketch of the devices shows the QSH edge states under the gate (shaded region). The ungated parts of the sample are $n$-type.

A $110 \mathrm{~nm}$ thick layer consisting of a $\mathrm{Si}_{3} \mathrm{~N}_{4} / \mathrm{SiO}_{2}$ superlattice is deposited on top of the structure by plasma-enhanced chemical vapor deposition (PECVD). This layer serves as a gate insulator between the semiconductor and the (Ti/Au) gate electrode on top of the structure. The top gate covers the leads of the Hall bar only to a small extent, while the largest parts of the leads are not gated, which implies that the mesa is always contacted by $n$-type HgTe leads. The gate insulator has excellent break-through properties and allows gate voltages in the range from $-5 \mathrm{~V}$ to $+5 \mathrm{~V}$ which is sufficient to induce a large change in carrier concentration [40,42]. However, in most structures not the full voltage swing can be applied. Hinz et al. have reported hysteresis effects in HgTe QWs at extreme gate voltages [43]. These effects are attributed to the filling and emptying of trap levels between the insulator and the semiconductor. In the experiments described below, care was taken to keep the gate voltages in the non-hysteretic regime. 


\section{High Field Characterization}

For the investigation of the QSH effect, samples with a low intrinsic density, i.e., $n\left(V_{g}=0\right)<5 \times 10^{11} \mathrm{~cm}^{-2}$, are studied. Transport measurements are done in a ${ }^{3} \mathrm{He} /{ }^{4} \mathrm{He}$-dilution refrigerator with a base temperature $T<30 \mathrm{mK}$ and a ${ }^{4} \mathrm{He}$ cryostat $(T=1.4 \mathrm{~K})$ fitted with a vector magnet system, which allows for magnetic fields up to $300 \mathrm{mT}$ in variable direction. When a negative gate voltage $V_{g}$ is applied to the top gate electrode of the device, the usual decrease of the electron density is observed. In Fig. $8 \mathrm{~A}$, measurements of the Hall resistance $R_{x y}$ are presented for a Hall bar with length $L=600 \mu \mathrm{m}$ and width $W=200 \mu \mathrm{m}$, respectively. The decrease of the carrier density is reflected in an increase of the Hall coefficient, when the gate voltage is lowered from $0 \mathrm{~V}$. For voltages down to $-1.3 \mathrm{~V}$, the density is decreased linearly from $3.5 \times 10^{11} \mathrm{~cm}^{2}$ to $0.5 \times 10^{11} \mathrm{~cm}^{2}$ (Fig. 8 B). For even lower gate voltages, the sample becomes
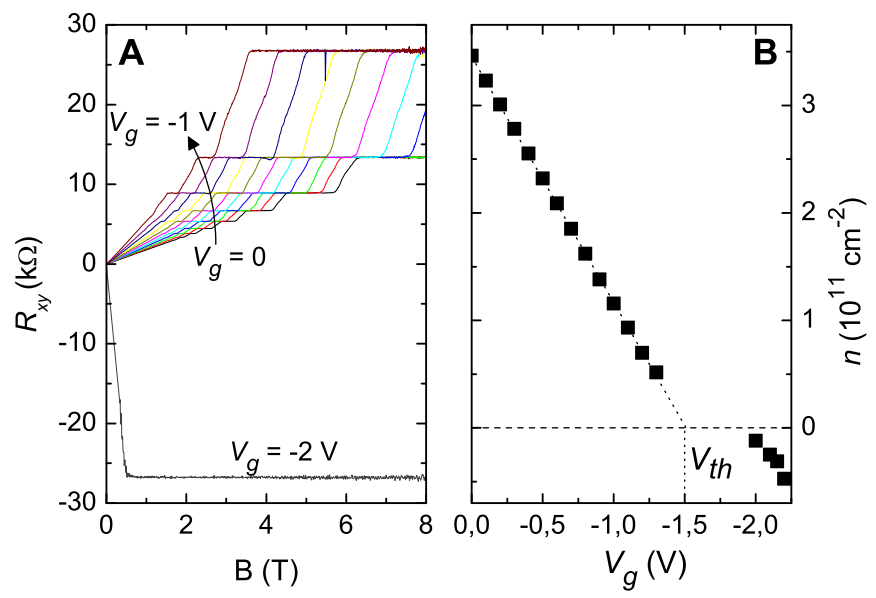

Figure 8: A: Hall resistance $R_{x y}$ as measured for various gate voltages, indicating the transition from $n$ - to $p$-conductance. B: The gate-voltage dependent carrier density deduced from the Hall measurements.

an insulator, with the Fermi level in the band gap. Remarkably, when a large negative voltage $V_{g} \leq-2 \mathrm{~V}$ is applied, the sample becomes conducting again. It can be inferred from the sign change of the Hall coefficient that the device is now $p$-conducting. Obviously, the Fermi level has now been pushed through the entire gap and into the valence band.

The transition from $n$ - to $p$-conductance is also reflected in the behavior of the longitudinal resistance $R_{x x}$ as a function of $V_{g}$ (cf. Fig. 113). For a decreasing electron density the resistance rises until it reaches a maximum when the Fermi level is in the gap. When the Fermi energy finally is pushed into the valence 
band and the sample is $p$-conducting, $R_{x x}$ decreases again by some orders of magnitude. A similar transition from $n$ - to $p$-conductance via an intermediate high resistance regime has been observed for a variety of QW structures with low intrinsic densities. The exact value of the maximum resistance, however, depends critically on the nature of the band structure, and thus on the actual QW width of the individual devices (cf. Section 7, below).

A similar signature in transport for a transition from $n$ - to $p$-conductance is possible and has been reported for the zero-gap material graphene [44]. However, since this material is a semi-metal, no insulating regime is observed between $n$ and $p$-conductance. Thus, the QSH effect is experimentally not accessible in graphene even though it was explicitly predicted for this material [11]. It was later understood theoretically that a possible energy gap in graphene due to spin-orbit coupling is far too small to be experimentally observable $[16,17]$.

The peculiar band structure of inverted $\mathrm{HgTe}$ quantum wells gives rise to a unique Landau level (LL) dispersion. For a normal band structure, i.e., $d_{Q W}<$ $d_{c}$, all Landau levels are shifted to higher energies for increasing magnetic field [Fig. 9 (a)]. This is the usual behavior and can be commonly observed for a variety of materials. When the band structure of the $\mathrm{HgTe} \mathrm{QW}$ is inverted
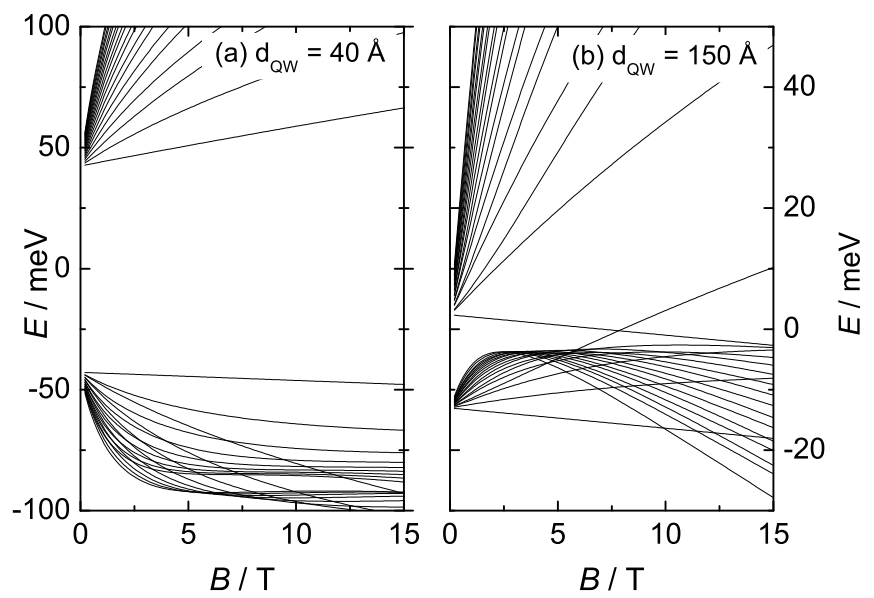

Figure 9: Landau level dispersion for (a) a $4.0 \mathrm{~nm}$ QW and (b) a $15.0 \mathrm{~nm}$ QW, respectively. The qualitative behavior is representative for samples with a normal and an inverted band structure, respectively.

$\left(d_{Q W}>d_{c}\right)$, the LL dispersion is markedly different [Fig. 9 (b)]. Due to the mixing of the electron-like and hole-like states, one of the states of the $H_{1}$ subband is a pure heavy-hole state $(M=-3 / 2)$. Consequently, the energy of the corresponding Landau level decreases with increasing magnetic field. In adition, one of the valence band LLs has mainly electron character and, thus, 
shifts to higher energies with magnetic field. This leads to a crossing of these two peculiar LLs for a finite magnetic field. The exact value of the crossing field $\mathcal{B}_{\text {cross }}$ depends on the QW width. Fig. [10] shows theoretically calculated $\mathcal{B}$-field

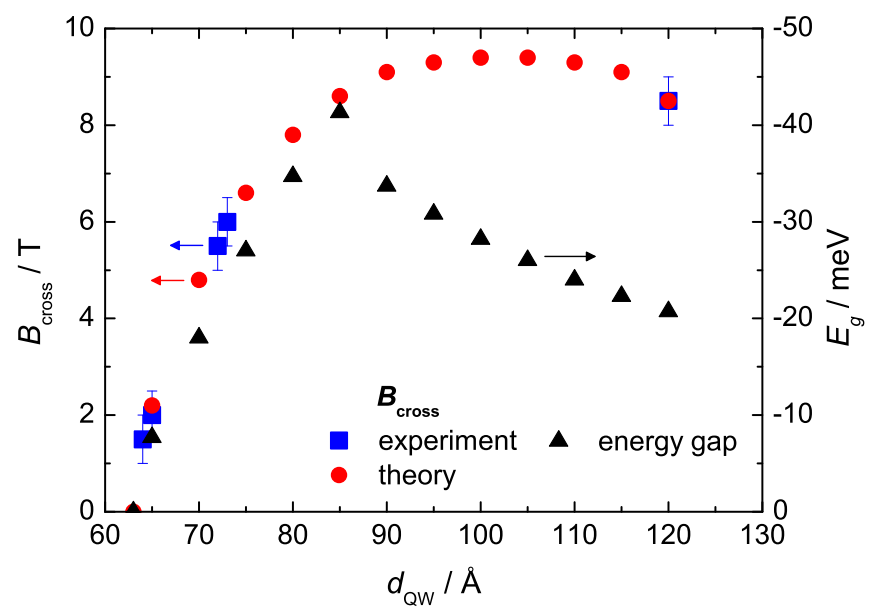

Figure 10: The $\mathcal{B}$-field value of the LL crossing determined experimentally (blue squares) and calculated theoretically (red circles). For the energy gap $E_{g}$ (black triangles), the negative values indicate the inverted band structure.

values for the Landau level crossing for quantum wells with an inverted band structure as red circles. The existence of such a LL crossing is a clear indication for the occurrence of an inverted band structure; for narrow QWs $\left(d_{Q W}<d_{c}\right)$ with a normal band structure, there is no LL crossing. The figure also plots the energy gap of the HgTe QWs, defined as $E_{1}-H_{1}$, as black triangles. In narrow wells $\left(d_{Q W}<d_{c}\right)$ with a normal band structure, our definition yields a positive gap, whilst for inverted wells we have an increasingly negative gap. For $d_{Q W} \approx 8.5 \mathrm{~nm}$, the $E_{1}$ subband falls below the $H_{2}$ band (cf. Fig. 2), and as a result the absolute value of the gap decreases subsequently for larger QW widths.

The crossing of the conduction and valence band Landau levels can be verified experimentally by quantum Hall experiments. Fig. 11] (a) shows quantum Hall data for an inverted quantum well with a width of $6.5 \mathrm{~nm}$. For gate voltages $V_{g} \geq-1.0 \mathrm{~V}$ and $V_{g} \leq-2.0 \mathrm{~V}$, the slope of the Hall signal directly shows that the Fermi level is firmly in the conduction and valence band, respectively. When the Fermi level is adjusted in the gap, i.e., $-1.9 \mathrm{~V}<V_{g}<-1.4 \mathrm{~V}$, the Hall resistance shows insulating behavior at zero field.

However, at higher magnetic fields the Hall resistance for these gate voltages exhibits a re-entrance of the $n=1$ quantum Hall plateau. This is a direct 


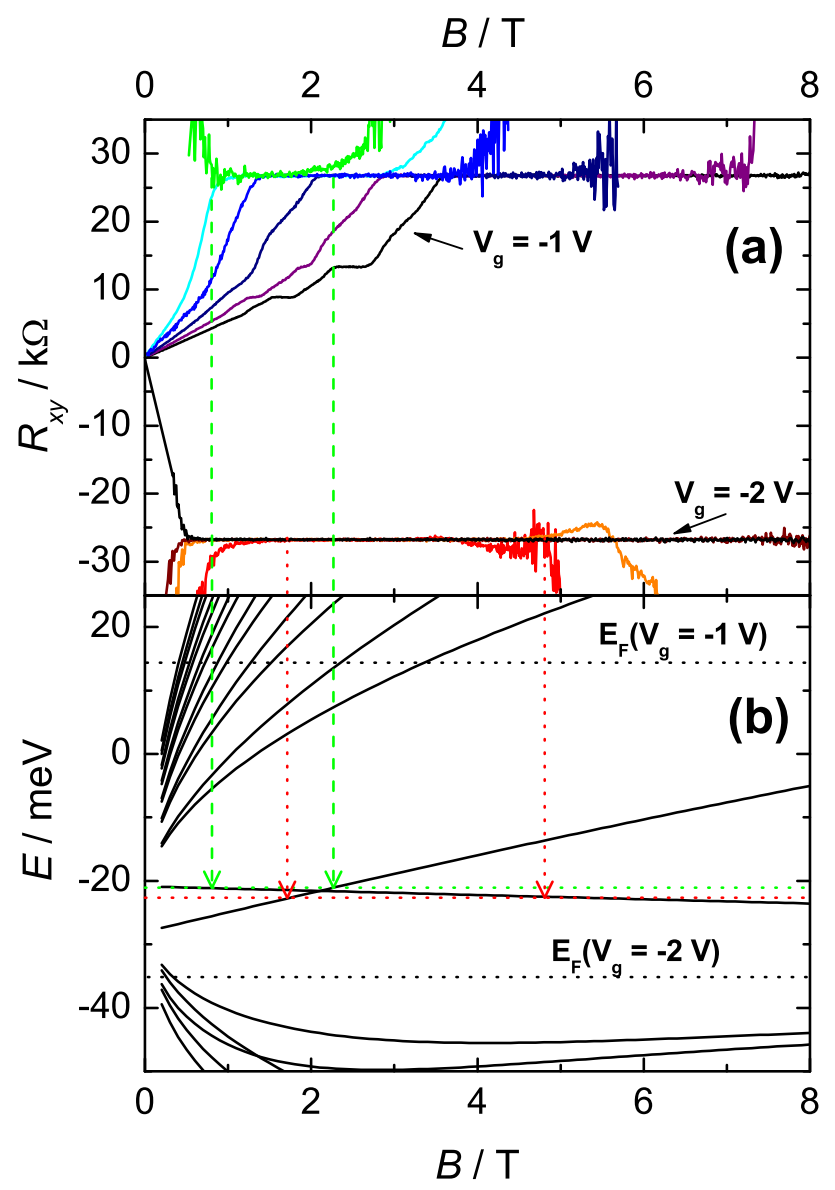

Figure 11: (a) Hall resistance, $R_{x y}$, of a $(L \times W)=(600 \times 200) \mu \mathrm{m}^{2}$ QW structure with $6.5 \mathrm{~nm}$ well width for different carrier concentrations obtained for different gate voltages $V_{g}$ in the range of $-1 \mathrm{~V}$ to $-2 \mathrm{~V}$. For decreasing $V_{g}$, the $n$-type carrier concentration decreases and a transition to a $p$-type conductor is observed, passing through an insulating regime between $-1.4 \mathrm{~V}$ and $-1.9 \mathrm{~V}$ at $\mathcal{B}=0 \mathrm{~T}$. (b) The Landau level fan chart of a $6.5 \mathrm{~nm}$ quantum well obtained from an eight band $\mathbf{k} \cdot \mathbf{p}$ calculation. Black dashed lines indicate the energetic position of the Fermi energy, $E_{F}$, for $V_{g}=-1.0 \mathrm{~V}$ and $-2.0 \mathrm{~V}$. Red and green dashed lines correspond to position of the Fermi energies of the red and green Hall resistance traces of (a). The crossing points of the Fermi level with the respective Landau levels are marked by arrows of the same color. 
consequence of the crossing of the Fermi level with the conduction-band derived LL for the green trace $\left(V_{g}=-1.4 \mathrm{~V}\right)$ and with the valence band LL (e.g, for the red trace where $V_{g}=-1.8 \mathrm{~V}$ ). As shown Fig. 11] (b), the experimental results are in good agreement with the theoretically calculated LL dispersion. The crossing point of the Landau levels in magnetic field, $\mathcal{B}_{\text {cross }}$, can be determined accurately by tuning the Fermi level through the energy gap. This method has been used for various samples with an inverted band structure; the results are included in Fig. 10 (blue squares).

The observation of a re-entrant quantum Hall state is a clear indication of the non-trivial insulating behavior, which is a prerequisite for the existence of the QSH state. In contrast, trivial insulating behavior is obtained for devices with $d_{Q W}<d_{c}$. For a normal band structure, the energy gap between the lowest Landau levels of the conduction band and the valence band, respectively, increases in magnetic field [cf. Fig. 9(a)]. Thus, a sample remains insulating in magnetic field, if the Fermi energy is located in the gap for $\mathcal{B}=0$.

The physics of this re-entrant quantum Hall state can basically be understood within the simple 4-band model. We will consider our model given in Eq. 6 expanded near $\mathbf{k}=0$ and in the presence of a uniform $\mathcal{B}$-field $(e \mathcal{B}>0)$ in the $z$-direction. We choose the symmetric gauge with $\mathbf{A}=\frac{\mathcal{B}}{2}(-y, x)$. We make the substitution $\mathbf{k} \rightarrow(\mathbf{k}+e \mathbf{A})$ and define new operators

$$
\begin{aligned}
& \pi_{+}=\hbar\left(k_{+}+\frac{i e \mathcal{B}}{2 \hbar} z\right) \\
& \pi_{-}=\hbar\left(k_{-}-\frac{i e \mathcal{B}}{2 \hbar} z^{*}\right)
\end{aligned}
$$

where $k_{ \pm}=\left(k_{x} \pm i k_{y}\right)$ and $z=x+i y$. These operators obey the commutation relations $\left[\pi_{+}, \pi_{-}\right]=\frac{-2 \hbar^{2}}{\ell_{\mathcal{B}}^{2}}$ with the magnetic length $\ell_{B}=(\hbar / e \mathcal{B})^{1 / 2}$. Using these commutation relations we can define raising and lowering operators

$$
\begin{gathered}
a=\frac{\ell_{B}}{\hbar} \pi_{-} \quad, \quad a^{\dagger}=\frac{\ell_{B}}{\hbar} \pi_{+} \\
{\left[a, a^{\dagger}\right]=1 .}
\end{gathered}
$$

Using these operators we rewrite our Hamiltonian as

$$
\begin{aligned}
& \mathcal{H}=\left(\begin{array}{cc}
h_{+}\left(a^{\dagger}, a\right) & 0 \\
0 & h_{-}\left(a^{\dagger}, a\right)
\end{array}\right) \\
& h_{+}\left(a^{\dagger}, a\right)=(C-D) \frac{2}{\ell_{B}^{2}}\left(a^{\dagger} a+\frac{1}{2}\right) I_{2 \times 2}+(M-B) \frac{2}{\ell_{B}^{2}}\left(a^{\dagger} a+\frac{1}{2}\right) \sigma^{z}+\frac{\sqrt{2} A}{\ell_{B}}\left(a^{\dagger} \sigma^{+}+a \sigma^{-}\right) \\
& h_{-}\left(a^{\dagger}, a\right)=(C-D) \frac{2}{\ell_{B}^{2}}\left(a^{\dagger} a+\frac{1}{2}\right) I_{2 \times 2}+(M-B) \frac{2}{\ell_{B}^{2}}\left(a^{\dagger} a+\frac{1}{2}\right) \sigma^{z} \\
&- \frac{\sqrt{2} A}{\ell_{B}}\left(a^{\dagger} \sigma^{-}+a \sigma^{+}\right)
\end{aligned}
$$


where $\sigma^{ \pm}=\frac{1}{2}\left(\sigma^{x} \pm i \sigma^{y}\right)$. It should be noted in this context that the materialspecific parameter $B$ of the Dirac model, which plays a crucial role in describing the re-entrant quantum Hall effect, is not related to the magnetic field. The spectrum of this Hamiltonian can be solved since only a finite number of harmonic oscillator Landau levels are coupled. We find the energy spectrum

$$
E_{\alpha}=-\omega_{0}^{D} n-\alpha \frac{\omega_{0}^{B}}{2} \pm \sqrt{\frac{2 A^{2} n}{\ell_{B}^{2}}+\left(M-n \omega_{0}^{B}-\frac{\alpha}{2} \omega_{0}^{D}\right)^{2}}
$$

where $\omega_{0}^{B}=\frac{2}{\ell_{B}^{2}} B, \omega_{0}^{D}=\frac{2}{\ell_{B}^{2}} D, \quad \alpha= \pm$ and $n=0,1,2, \ldots$ This spectrum has "zero modes" given by

$$
\begin{aligned}
& E_{+}^{0}=-M-\frac{1}{2}\left(\omega_{0}^{B}-\omega_{0}^{D}\right) \\
& E_{-}^{0}=M+\frac{1}{2}\left(\omega_{0}^{B}+\omega_{0}^{D}\right)
\end{aligned}
$$

for $M / 2 B<0$ and

$$
\begin{aligned}
& E_{+}^{0}=M-\frac{1}{2}\left(\omega_{0}^{B}+\omega_{0}^{D}\right) \\
& E_{-}^{0}=-M+\frac{1}{2}\left(\omega_{0}^{B}+\omega_{0}^{D}\right)
\end{aligned}
$$

for $M / 2 B>0$. For $M / 2 B<0$ one can see that the set of zero-modes never cross. However, for $M / 2 B>0$, i.e., in the inverted QSH regime, the zero modes do cross at a field of $\mathcal{B}_{\text {cross }}=\frac{\hbar}{e} \frac{M}{B+D}$. For typical parameters in the inverted regime $[18,45]$ the critical field is just a few Tesla. Since the Dirac mass $M$ reflects the energy gap, the linear dependence of $\mathcal{B}_{\text {cross }}$ on $M$ in the 4-band Dirac model is in good agreement with the results obtained by the $8 \times 8$ Hartree calculations shown in Fig. 10. The typical Landau level spectra for the non-inverted and inverted regime, respectively, presented in Fig. 12 (a),(b) are obtained by the simple 4-band model.

As illustrated in Fig. 12 there are four different regimes of interest. Two have quantized Hall conductances of $\pm e^{2} / h$ and the other two have vanishing Hall conductances. Case (i) is described by $\mathcal{B}>\mathcal{B}_{c}$, while the chemical potential is placed between the zero modes. Here no edge states exist and this yields $\sigma_{x y}=0$. Identical behavior is obtained for a trivial insulator independent of the $\mathcal{B}$-field strength, if the chemical potential is in the energy gap. Case (ii) also shows $\sigma_{x y}=0$. However, there should be counter-propagating edge states that carry opposite Hall current, giving rise to the QSH effect. When the chemical potential is slightly below the two zero modes as in case (iii), only one of them will provide edge states. Thus, there is a single state at each edge, resembling the well-known quantum Hall regime with $\sigma_{x y}=e^{2} / h$. A similar result is obtained for case (iv), where the chemical potential is just below the zero modes. However, since the single edge state now originates from the other zero mode, the Hall conductance changes sign, i.e., $\sigma_{x y}=-e^{2} / h$. If the chemical potential 
is fixed and unequal to the energy of the LL crossing $E\left(\mathcal{B}_{\text {cross }}\right)$, an increase of the magnetic field gives rise to the re-entrant quantum Hall state. While the Hall conductance vanishes for case (i) and (ii), an intermediate regime with $\sigma_{x y}= \pm e^{2} / h$ according to case (iii) and (iv), respectively, will be obtained, while the sign of $\sigma_{x y}$ depends on the exact chemical potential.

\section{Experimental observation of the QSH effect at $\mathcal{B}=0$}

The existence of the QSH effect is revealed when small Hall bars $(L \times W=$ $20.0 \mu \mathrm{m} \times 13.3 \mu \mathrm{m})$ are studied. For QW devices with a normal band structure, the sample shows trivial insulating behavior (Fig. 13). The resistance of several $\mathrm{M} \Omega$ for the insulating regime can be attributed to the intrinsic noise level of the measurement setup, so that the conductance basically vanishes. For a device with an inverted band structure, however, the resistance stays finite, not exceeding $100 \mathrm{k} \Omega$. This behavior is reproduced for various Hall bars with a QW width in the range from $4.5 \mathrm{~nm}$ to $12.0 \mathrm{~nm}$ : while devices with a normal band structure, i.e., $d_{Q W}<d_{c} \approx 6.3 \mathrm{~nm}$, show trivial insulating behavior, a finite conductance in the insulating regime is observed for samples with an inverted band structure. We now proceed ro relate this observed finite conductance to the predicted edge state behavior. In the theoretical modelling of Ref. 18, ideal contacts to the QSH edge states were assumed, i.e., the potential of the edge states can be probed without any influence due to the contacts. However, in reality the leads are always $n$-type for the investigated samples and remain such independently of the applied gate voltage. Unlike for a quantum Hall system, the edge states in a QSH system propagate in both directions at a given edge (cf. Fig. 7). Thus, the edge states entering a contact carry a different potential due to the different sources. When the edge states enter an $n$-type contact, they necessarily equilibrate with the bulk states and backscattering becomes possible. Hence, the voltage difference between neighboring contacts does not vanish even though they are connected by non-dissipative edge channels. In other words, a voltage probe induces additional resistance, even when no voltage is applied to it. Such a dissipation occurs due to the large size of the $n$-type contact, which implies a huge number of channels the incoming electron can scatter to. Consequently, an infinitesimal dissipation in the contact can cause complete decoherence between the incoming and outgoing electrons. This analysis can be easily confirmed by a Green's function calculation of the ballistic conductance in the Keldysh formulism. In contrast, in a quantum Hall system, a voltage probe does not induce additional dissipation, because all the states are propagating to the same direction. The de-coherence effect of a contact, although also it exists, does not affect the current through it.

A simple Landauer-Büttiker formalism [46] can be applied to determine the influence of the $n$-type contacts. The current $I_{i}$ in a contact $i$ (cf. Fig. 7) can 
(A)

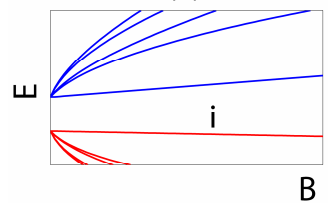

(C)
(B)

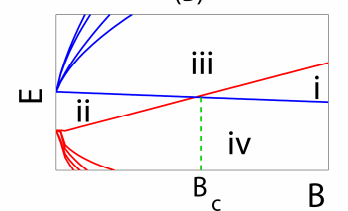

(D)

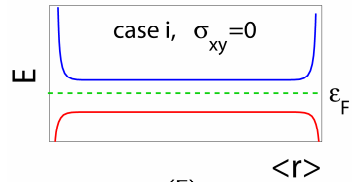

(E)

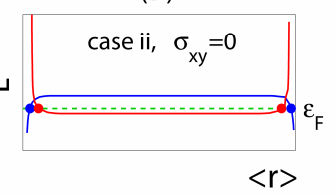

(F)

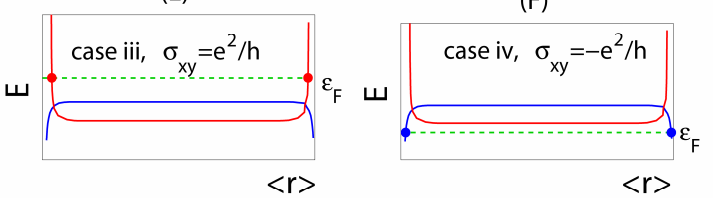

Figure 12: (a) Landau level fan diagram for the $E 1$ and $H 1$ subbands in the noninverted regime. (b)Landau level fan diagram for the $E 1$ and $H 1$ subbands in the inverted regime. Notice that the zero-modes from each set of subband Landau levels cross at a finite $\mathcal{B}$-field. Lower case roman numerals indicate separate regions in energy and magnetic field. (c)-(f)Schematic energy spectrum of the zero-modes and edge states for region i,ii,iii, and iv respectively. (c)Region i produces a vanishing hall-conductance. (d) Region ii produces a vanishing hall-conductance but the Fermi-level passes through two branches of quantum Hall edge states which are oppositely circulating and carry opposite current. (e) Region iii produces a Hall-conductance of $+e^{2} / h$, and the Fermi-level only crosses one of the sets of edge states. (f) Region iv produces a Hall-conductance of $-e^{2} / h$, and the Fermi-level only crosses one of the sets of edge states. 


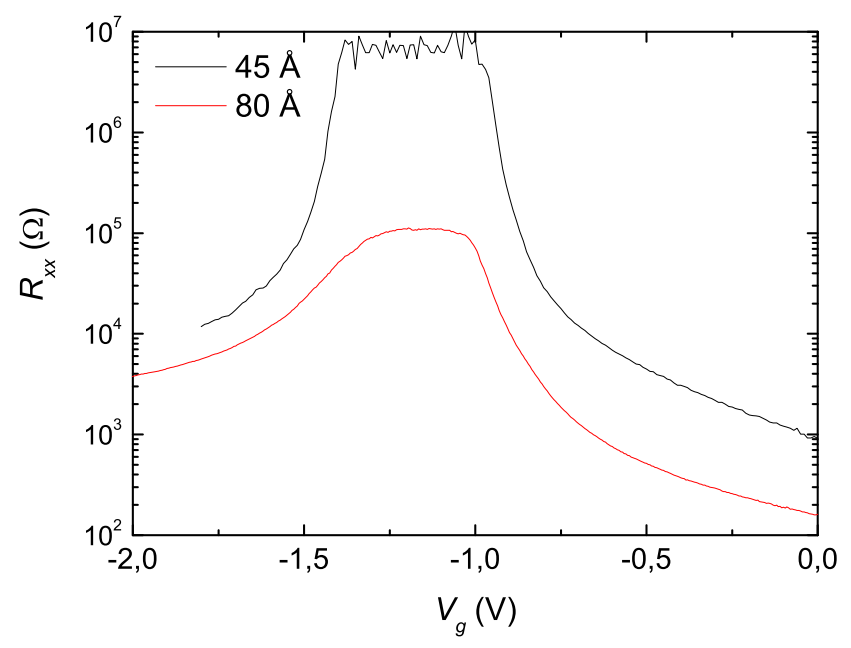

Figure 13: The longitudinal resistance of a $4.5 \mathrm{~nm}$ QW (a) and an $8.0 \mathrm{~nm}$ QW (b) as a function of gate voltage, respectively.

be calculated from

$$
I_{i}=(e / h) \sum_{j} T_{i j}\left(\mu_{j}-\mu_{i}\right) .
$$

Since the edge states are non-interacting, the transmission coefficient $T_{i j}$ is unity for neighboring contacts and zero otherwise. In the measurements, a current $I$ is injected from contact 1 to contact 4 , i.e., $I_{1}=-I_{4}=I$, while $I_{i}=0$ vanishes for all other contacts serving as voltage probes. When total equilibration of all edge states in each contact is assumed, a four-terminal resistance for neighboring voltage probes of $\left(h / 2 e^{2}\right)$ is obtained. The two-terminal resistance is determined by the number $n$ of the voltage probes between the current source and drain. Between each pair of neighboring contacts, a voltage $V_{i}=I \cdot\left(h / e^{2}\right)$ drops, adding up to a total voltage drop of $(n+1) V_{i}$. Thus, the two-terminal resistance is $(3 h) /\left(2 e^{2}\right)$ for a Hall bar geometry with two voltage probes on each side as shown in Fig. 7

However, the obtained finite resistance due to the $n$-type contacts still does not give a quantitative explanation for the observed resistance $R \approx 100 \mathrm{k} \Omega$, which is significantly higher than four terminal resistance $\left(h / 2 e^{2}\right) \approx 12.9 \mathrm{k} \Omega$ one anticipates for the geometry used in the experiments. The enhanced resistance in these samples with a length of $L=20 \mu \mathrm{m}$ can be readily understood when inelastic scattering is considered. While, as discussed above, the helical edge states are robust against single-particle elastic backscattering, inelastic mechanisms can cause backscattering. 
For $n$-doped $\mathrm{HgTe}$ quantum wells, the typical mobility of the order $10^{5} \mathrm{~cm}^{2} / \mathrm{Vs}$ implies an elastic mean free path of the order of $1 \mu \mathrm{m}$ [41]. Lower mobilities can be anticipated for the QSH regime. The inelastic mean free path, which determines the length scale of undisturbed transport by the QSH edge states, can be estimated to be several times larger due to the suppression of phonons and the reduced electron-electron interaction at low temperatures. Thus, the inelastic scattering length is of the order of a few microns.

For the observation of the QSH conductance, the sample dimensions were reduced below the estimated inelastic mean free path. When Hall bars with a length $L=1 \mu \mathrm{m}$ are studied, a four-terminal resistance close to $h /\left(2 e^{2}\right)$ is observed. The threshold voltage $V_{t h}$ is defined such that the QSH regime is in the vicinity of $V_{g}=V_{t h}$. The slight deviation of $R$ from the quantized value

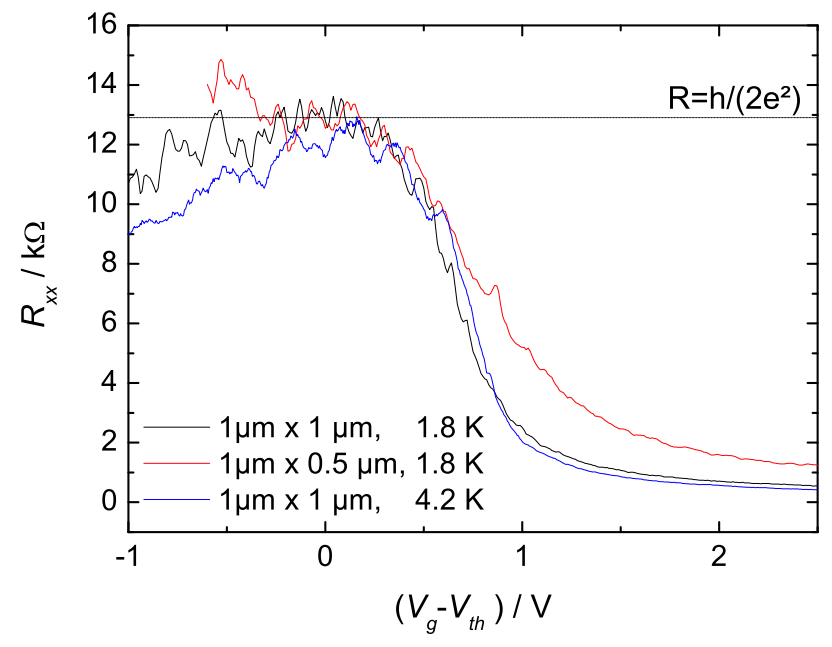

Figure 14: The longitudinal resistance is measured as a function of the gate voltage for two devices with $L=1 \mu \mathrm{m}$. The width $W$ is $1 \mu \mathrm{m}$ (black and blue) and $0.5 \mu \mathrm{m}$ (red), respectively. The black and red trace were obtained at $1.8 \mathrm{~K}$, the blue one at $4.2 \mathrm{~K}$.

$h /\left(2 e^{2}\right)$ can be attributed to some residual scattering. This is an indication that the length of the edge states still exceeds the inelastic mean free path. However, a significant increase of the conductance is obtained by reducing the sample size, which demonstrates clearly the effect of scattering on the transport by QSH states.

The results presented in Fig. 14 provide evidence that transport in the QSH regime indeed occurs due to edge states. The two devices with $W=1.0 \mu \mathrm{m}$ and $W=0.5 \mu \mathrm{m}$, respectively, were fabricated from the same QW structure. The resistance values of the two devices differ significantly for the $n$-conducting 
regime, where transport is determined by bulk properties. For the insulating regime, however, both devices show the same resistance, even though the width of the devices differs by a factor 2 . For all three measurements, no full transition to the $p$-conducting regime can be observed. This is attributed to charging of the quantum well layer during the e-beam process required to fabricate such small samples. The fluctuations, which are observable in all traces, are reproducible. Thus, they must have a physical reason, which is not fully clear yet, and can not be caused, e.g., by electrical noise. The pure QSH signal, however, is robust against an increase of temperature as long as $k_{B} T$ is much smaller than the two-dimensional bulk energy gap.

\section{Suppression of the quantum spin Hall effect by a magnetic field}

Further clear evidence for the QSH effect is obtained by measurements in a finite magnetic field, which destroys the time reversal symmetry of the QSH states. In order to observe this effect, the dependence of the QSH conductance is studied in a magnetic field in various directions with respect to the plane of the 2DEG. For this purpose an inverted QW in Hall bar geometry with $(L \times W)=(20.0 \times 13.3) \mu \mathrm{m}^{2}$ was investigated at $1.4 \mathrm{~K}$ in a vector magnet system. In a perpendicular field configuration the QSH conductance decreases rapidly when the magnetic field is applied. A cusp-like conductance peak is observed with a full width-half maximum field $\mathcal{B}_{\text {FWHM }}$ of $28 \mathrm{mT}$ (Fig. 15), which is even smaller at lower temperatures. At $T=30 \mathrm{mK}$ a typical width of $\mathcal{B}_{\mathrm{FWHM}}=10 \mathrm{mT}$ is observed. When the magnetic field direction is rotated into the plane of the 2DEG the conductance peak widens strongly, reaching a value of $\mathcal{B}_{\mathrm{FWHM}} \approx 0.7 \mathrm{~T}$ for the parallel configuration.

From the results shown in Fig. 15] it is evident that a perpendicular field has a much larger influence on the helical liquid than an in-plane field.

Such a large anisotropy can be understood by a slightly modified version of the four-band effective model we discussed in Sec. 3. To first order in perturbation theory, the gap induced by a perpendicular and an in-plane field can be determined by the matrix elements of the corresponding operator:

$$
\begin{aligned}
E_{\text {gap } \perp} & =\left|\left\langle k=0,+\left|\left(\hat{\mathbf{z}} \cdot \overrightarrow{\mathbf{r}} \times \overrightarrow{\mathbf{j}}+\mu_{B} M_{z}\right) \mathcal{B}_{\perp}\right| k=0,-\right\rangle\right| \\
E_{\text {gap } \|} & =\left|\left\langle k=0,+\left|\mu_{B}\left(M_{x} \mathcal{B}_{x}+M_{y} \mathcal{B}_{y}\right)\right| k=0,-\right\rangle\right|
\end{aligned}
$$

where $\overrightarrow{\mathbf{r}}, \overrightarrow{\mathbf{j}}$ are electron position and current operators, respectively, and $\hat{\mathbf{z}}$ is the unit-vector perpendicular to the 2DEG plane. The states $|k=0, \pm\rangle$ are the two edge states propagating toward opposite directions on the same boundary. The Zeeman coupling matrix elements $M_{x, y, z}$ in the four-band effective model can be determined by standard perturbation procedures from the original Kane 


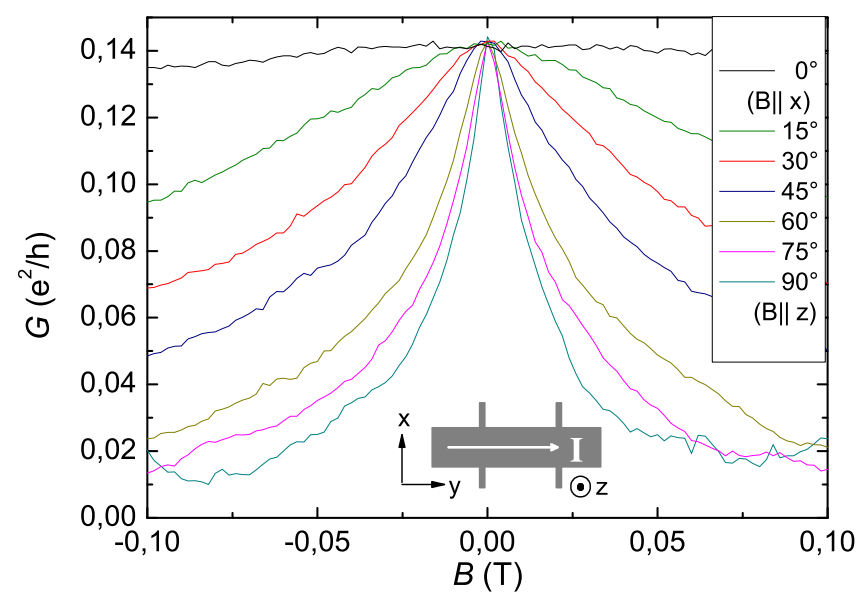

Figure 15: Four-terminal magnetoconductance, $G_{14,23}$, in the QSH regime as a function of tilt angle between the plane of the 2DEG and applied magnetic field for a $d=7.3 \mathrm{~nm}$ QW structure with dimensions $(L \times W=20 \times 13.3) \mu \mathrm{m}^{2}$ measured in a vector field cryostat at $1.4 \mathrm{~K}$.

model, which has the form [45]

$$
\begin{aligned}
& M_{z}=\left(\begin{array}{llll}
g_{E \perp} & & & \\
& g_{H \perp} & & \\
& & -g_{E \perp} & \\
& & & -g_{H \perp}
\end{array}\right) \\
& M_{x}=\left(\begin{array}{lll} 
& g_{\|} \\
& & \\
g_{\|} &
\end{array}\right), M_{y}=\left(\begin{array}{ll} 
& -i g_{\|} \\
i g_{\|} &
\end{array}\right) .
\end{aligned}
$$

Since the edge state wavefunctions of the effective model have been obtained in Sec. 3, the gaps $E_{\text {gap } \perp}$ and $E_{\text {gap } \|}$ can be calculated for given $g$-factors and Zeeman couplings $S_{x, y, z}$. However, there is a subtlety that has to be considered before carrying out this calculation. The effective model (Eq. 1) consists of two decoupled blocks, which means the coupling between $|E 1,+\rangle,|H 1,+\rangle$ and $|E 1,-\rangle,|H 1,-\rangle$ is ignored. Thus for such an effective model, the current operator $\overrightarrow{\mathbf{j}}=\nabla_{\mathbf{k}} H(\mathbf{k})$ is also block diagonal. Since the wavefunction of the edge state $|k,+(-)\rangle$ consists only $|E 1,+(-)\rangle$ and $|H 1,+(-)\rangle$ components, the orbital magnetization operator $\hat{\mathbf{z}} \cdot \overrightarrow{\mathbf{r}} \times \overrightarrow{\mathbf{j}}$ has a vanishing matrix element between them, which means the orbital effect of the magnetic field does not contribute to the

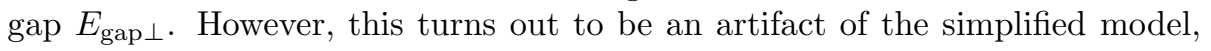


which comes from the absence of bulk inversion asymmetry (BIA) terms. When the breaking of bulk inversion symmetry is taken into account, an additional term is induced in the four-band effective model [45]:

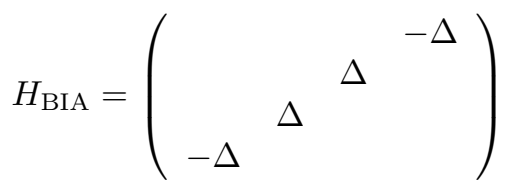

which describes the mixing between $|E 1,+(-)\rangle$ and $|H 1,-(+)\rangle$. Such a term preserves time-reversal symmetry, and does not close the bulk gap, and thus does not affect the topological stability of the nontrivial insulator phase. Consequently, all the theoretical discussions based on the four-band model that we presented in the earlier part of the paper are only slightly modified. Nevertheless, such a term becomes important when we consider the effect of perpendicular magnetic fields. Since the bulk-inversion symmetric terms have a vanishing contribution to the edge state gap, the contribution from BIA terms becomes the leading order. The BIA term $\Delta$ and all other parameters $A, B, C, D, M, g_{E \perp}, g_{H \perp}, g_{\|}$in the model can be obtained from envelope function calculations of the quantum well [45]. For the quantum well thickness $d=7.0 \mathrm{~nm}$, these parameters are given as

\begin{tabular}{|c|c|c|c|c|c|c|c|}
\hline 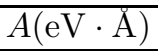 & $B\left(\mathrm{eV} \cdot \AA^{2}\right)$ & $D(\mathrm{eV})$ & $M(\mathrm{eV})$ & $\Delta(\mathrm{eV})$ & $g_{E \perp}$ & $g_{H \perp}$ & $g_{\|}$ \\
\hline 3.645 & -68.6 & -51.2 & -0.010 & 0.0016 & 22.7 & -1.21 & -20.5 \\
\hline
\end{tabular}

By inputing these parameters into the tight-binding model (Eq. 6) the edge state wavefunction can be obtained numerically. For a magnetic field of 1T, the gap induced is $E_{\text {gap } \perp}=3.1 \mathrm{meV}$ if it is perpendicular to the 2DEG plane, or $E_{\text {gap } \|}=0.3 \mathrm{meV}$ if parallel. Since the perpendicular and in-plane Zeeman coupling are of the same order, we see that the main contribution of $E_{\text {gap } \perp}$ comes from the orbital effect of the magnetic field. Compared to the Zeeman contribution, the orbital effect is equivalent to an "effective $g$-factor" of order $10^{2}$. Besides the numerical calculations, the reason for such a large orbital effect can also be understood by a rough order-of-magnitude estimation. Since the current operator still doesn't mix $|E(H),+\rangle$ and $|E(H),-\rangle$ even with BIA terms in the Hamiltonian, the only contribution to $E_{\text {gap }} \perp$ comes from the weight of $|E(H),-\rangle$ components in the edge state wavefunction $|k=0,+\rangle$, which is proportional to $\Delta /|M|$ from first order perturbation theory. Thus we can estimate the matrix element of the orbital magnetization operator as $\langle k=0,+|\hat{\mathbf{z}} \cdot \overrightarrow{\mathbf{r}} \times \overrightarrow{\mathbf{j}}| k=0,-\rangle \sim(\Delta /|M|) e v_{F} \xi$, where we use $e v_{F}$ and the edge state width $\xi$ as the estimates of operators $\overrightarrow{\mathbf{j}}$ and $\overrightarrow{\mathbf{r}}$, respectively. $v_{F}$ and $\xi$ of the edge states can be estimated by $v_{F}=A / \hbar, \xi \simeq A /|M|$. Thus $E_{\text {orb }} \sim \frac{e}{\hbar} \frac{\Delta A^{2}}{M^{2}}\left|\mathcal{B}_{\perp}\right|$. Compared with the Bohr magnon we have the "effective $g$-factor" as

$$
g_{\mathrm{eff}} \sim \frac{e}{\hbar} \frac{\Delta A^{2}}{M^{2}} \frac{1}{\mu_{B}}=\frac{2 m_{e} v_{F}^{2}}{M^{2} / \Delta}
$$


Since $v_{F}=A / \hbar \simeq 5.5 \times 10^{5} \mathrm{~m} / \mathrm{s}$, we obtain $g_{\text {eff }}$ is around 50 . Such an analysis also leads to the prediction that the anisotropy between perpendicular and inplane magneto-conductance is further enhanced when the thickness goes closer to the critical thickness $d_{c}$ from the inverted side, due to the decrease of $|M|$.

\section{Conclusion}

We have reviewed our current theoretical understanding of the QSH state, with a focus on the experimental realization in $\mathrm{HgTe}$ quantum wells. We discussed the electronic structure of the $\mathrm{HgTe}$ quantum wells in terms of a simple 4 band model which contains the essential physics. We demonstrated the topological quantum phase transition as the quantum well thickness $d_{Q W}$ is varied and we explicitly showed the analytic solution of the helical edge states on the topologically nontrivial side of the phase transition. We discussed the topological stability of the helical edge states using the concrete example of the 4 band model for $\mathrm{HgTe}$ quantum wells and presented the experimental realization of the QSH effect in $\mathrm{HgTe}$ QW structures for various QW widths. These experiments clearly demonstrated the edge channel character of the QSH effect and that the inverted band structure, which occurs for HgTe QW with $d_{Q W}>6.3 \mathrm{~nm}$, is essential. Deviation from the quantized conduction value for large samples and the effect of an applied magnetic field could be related to back-scattering introduced by time-reversal symmetry breaking processes.

The actual experimental realization of the QSH effect opens up the opportunity for investigations of new theoretical concepts [47], concerning fundamental aspects and applications, utilizing the spin polarized properties of the helical edge channels.

We wish to thank B.A. Bernevig, X. Dai, Z. Fang and C.J. Wu for insightful discussions, S. Wiedmann for assistance with the experiments, A. Roth, C. Brüne, C.R. Becker and V. Hock for sample preparation, and C. Kumpf for calibrating the well widths of the HgTe samples. This work is supported by the DFG (SFB 410), by the German-Israeli Foundation for Scientific Research and Development (Grant No.881/05), by NSF through the grants DMR-0342832, and by the US Department of Energy, Office of Basic Energy Sciences under contract DE-AC03-76SF00515, and Focus Center Research Program (FCRP) Center on Functional Engineered Nanoarchitectonics (FENA).

\section{References}

[1] G. Prinz. Science, 282:1660, 1998.

[2] S. A. Wolf et. al. Science, 294:1488, 2001.

[3] S. Murakami, N. Nagaosa, and S.C. Zhang. 301:1348, 2003.

[4] J. Sinova et. al. Phys. Rev. Lett., 92:126603, 2004. 
[5] Y. Kato et. al. Nature, 427:50, 2004.

[6] J. Wunderlich, B. Kaestner, J. Sinova, and T. Jungwirth. Phys. Rev. Lett., 94:47204, 2005.

[7] M.I. Dyakonov and V.I. Perel. Sov. Phys. JETP, 13:467, 1971.

[8] J.E. Hirsch. Phys. Rev. Lett., 83:1834, 1999.

[9] D.J. Thouless, M. Kohmoto, M.P. Nightingale, and M. den Nijs. Phys. Rev. Lett., 49:405, 1982.

[10] S. Murakami, N. Nagaosa, and S.C. Zhang. Phys. Rev. Lett., 93:156804, 2004.

[11] C. L. Kane and E. J. Mele. Phys. Rev. Lett., 95:226801, 2005.

[12] B.A. Bernevig and S.C. Zhang. Phys. Rev. Lett., 96:106802, 2006.

[13] C. Wu, B.A. Bernevig, and S.C. Zhang. Phys. Rev. Lett., 96:106401, 2006.

[14] C. Xu and J. Moore. Phys. Rev. B, 73:045322, 2006.

[15] C. L. Kane and E. J. Mele. Phys. Rev. Lett., 95:146802, 2005.

[16] Yugui Yao, Fei Ye, Xiao-Liang Qi, Shou-Cheng Zhang, and Zhong Fang. Phys. Rev. B, 75:041401(R), 2007.

[17] H. Min, J. Hill, N. Sinitsyn, B. Sahu, L. Kleinman, and A. MacDonald. Phys. Rev. B, 74:165310, 2006.

[18] B. A. Bernevig, T. L. Hughes, and S.C. Zhang. Science, 314:1757, 2006.

[19] E. G. Novik, A. Pfeuffer-Jeschke, T. Jungwirth, V. Latussek, C. R. Becker, G. Landwehr, H. Buhmann, and L. W. Molenkamp. Phys. Rev. B, 72:035321, 2005 .

[20] A. Pfeuffer-Jeschke. PhD thesis, Universität Würzburg, 2000.

[21] E.O. Kane. J. Phys. Chem. Solids, 1:249, 1957.

[22] A. N. Redlich. Phys. Rev. D, 29:2366, 1984.

[23] G. Volovik. The Universe in a Helium Droplet. Oxford Publications, Oxford, 2003.

[24] X.L. Qi, Y.S. Wu, and S.C. Zhang. Phys. Rev. B, 74:085308, 2006.

[25] M. Creutz and I. Horvath. Phys. Rev. D, 50:2297, 1994.

[26] M. Creutz. Rev. Mod. Phys., 73:119, 2001.

[27] X. G. Wen. Phys. Rev. B, 41:12838, 1990. 
[28] H. B. Nielsen and M. Ninomiya. Nucl. Phys. B, 185:20, 1981.

[29] H. B. Nielsen and M. Ninomiya. Nucl. Phys. B, 193:173, 1981.

[30] Liang Fu and C. L. Kane. Phys. Rev. B, 74(19):195312, 2006.

[31] Liang Fu and C. L. Kane. Phys. Rev. B, 76(4):045302, 2007.

[32] Liang Fu, C. L. Kane, and E. J. Mele. Phys. Rev. Lett., 98(10):106803, 2007.

[33] J. E. Moore and L. Balents. Phys. Rev. B, 75:121306, 2007.

[34] Rahul Roy. arxiv: cond-mat/0604211.

[35] Rahul Roy. arxiv: cond-mat/0607531.

[36] Rahul Roy. arxiv: cond-mat/0608064.

[37] Qian Niu, D. J. Thouless, and Yong-Shi Wu. Phys. Rev. B, 31:3372, 1985.

[38] Andrew M. Essin and J. E. Moore. Physical Review B, 76:165307, 2007.

[39] Sung-Sik Lee and Shinsei Ryu. arxiv:0708.1639.

[40] C. R. Becker, C. Brüne, M. Schäfer, A. Roth, H. Buhmann, and L. W. Molenkamp. phys. stat. sol. (c), 4:3382, 2007.

[41] V. Daumer, I. Golombek, M. Gbordzoe, E. G. Novik, V. Hock, C. R. Becker, H. Buhmann, and L. W. Molenkamp. Appl. Phys. Lett., 83:1376, 2003.

[42] X. C. Zhang, A. Pfeuffer-Jeschke, K. Ortner, V. Hock, H. Buhmann, C. R. Becker, and G. Landwehr. Phys. Rev. B, 63:245305, 2001.

[43] J. Hinz, H. Buhmann, M. Schäfer, V. Hock, C R. Becker, and L. W. Molenkamp. Semicond. Sci. Technol., (21):501, 2006.

[44] A. K. Geim and K. S. Novoselov. Nature Materials, 6:183, 2007. and references therein.

[45] Taylor L. Hughes, Chao-Xing Liu, Xiao-Liang Qi, and Shou-Cheng Zhang. in preparation.

[46] M. Büttiker. Phys. Rev. Lett., 57:1761, 1986.

[47] Xiao-Liang Qi, Taylor L. Hughes, and Shou-Cheng Zhang. arxiv:0710.0730. 